This is an electronic reprint of the original article. This reprint may differ from the original in pagination and typographic detail.

Author(s): Kemppainen, Jukka; Siljander, Juhana; Vergara, Vicente; Zacher, Rico

Title: Decay estimates for time-fractional and other non-local in time subdiffusion equations in $R^{\wedge} d$

Year: $\quad 2016$

Version:

Please cite the original version:

Kemppainen, J., Siljander, J., Vergara, V., \& Zacher, R. (2016). Decay estimates for time-fractional and other non-local in time subdiffusion equations in $\mathrm{R}^{\wedge} \mathrm{d}$.

Mathematische Annalen, 366(3), 941-979. https://doi.org/10.1007/s00208-0151356-z

All material supplied via JYX is protected by copyright and other intellectual property rights, and duplication or sale of all or part of any of the repository collections is not permitted, except that material may be duplicated by you for your research use or educational purposes in electronic or print form. You must obtain permission for any other use. Electronic or print copies may not be offered, whether for sale or otherwise to anyone who is not an authorised user. 


\title{
Decay estimates for time-fractional and other non-local in time subdiffusion equations in $\mathbb{R}^{d}$
}

\author{
Jukka Kemppainen*, Juhana Siljander $^{\dagger}$, Vicente Vergara ${ }^{\ddagger}$, and Rico Zacher ${ }^{\S}$
}

\begin{abstract}
We prove optimal estimates for the decay in time of solutions to a rather general class of non-local in time subdiffusion equations in $\mathbb{R}^{d}$. An important special case is the timefractional diffusion equation, which has seen much interest during the last years, mostly due to its applications in the modeling of anomalous diffusion processes. We follow three different approaches and techniques to study this particular case: (A) estimates based on the fundamental solution and Young's inequality, (B) Fourier multiplier methods, and (C) the energy method. It turns out that the decay behaviour is markedly different from the heat equation case, in particular there occurs a critical dimension phenomenon. The general subdiffusion case is treated by method (B) and relies on a careful estimation of the underlying relaxation function. Several examples of kernels, including the ultraslow diffusion case, illustrate our results.
\end{abstract}

AMS subject classification: $35 \mathrm{R} 11,45 \mathrm{~K} 05,47 \mathrm{G} 20$

Keywords: temporal decay estimates, time-fractional diffusion, ultraslow diffusion, subdiffusion, fundamental solution, subordination, Fourier multiplier, energy estimates

\section{Introduction and main results}

The main purpose of this paper is to study the temporal decay of solutions to non-local in time diffusion equations whose prototype is given by

$$
\partial_{t}\left(k *\left[u-u_{0}\right]\right)-\Delta u=0, \quad t>0, x \in \mathbb{R}^{d},
$$

together with initial condition

$$
\left.u\right|_{t=0}=u_{0}, x \in \mathbb{R}^{d} .
$$

Here $u_{0}(x)$ is a given datum. $k * v$ denotes the convolution on the positive halfline $\mathbb{R}_{+}:=[0, \infty)$ w.r.t. the time variable, that is $(k * v)(t)=\int_{0}^{t} k(t-\tau) v(\tau) d \tau, t \geq 0$. Note that for sufficiently smooth $u$ satisfying (2),

$$
\partial_{t}\left(k *\left[u-u_{0}\right]\right)=k * \partial_{t} u .
$$

The kernel $k$ belongs to a wide class of kernels, it is merely assumed to satisfy the condition

* J.K., J.S. and R.Z. were partially supported by Academy of Finland project 138738 (Nonlinear PDEs, project lead by professor Juha Kinnunen)

${ }^{\dagger}$ J.S. was supported by Academy of Finland postdoctoral research grant 259363

$\ddagger$ V.V. was partially supported by FONDECYT grant 1150230 .

${ }_{\S}$ R.Z. was supported by a Heisenberg fellowship of the German Research Foundation (DFG), GZ Za 547/3-1. 
$(\mathcal{P C}) k \in L_{1, \text { loc }}\left(\mathbb{R}_{+}\right)$is nonnegative and nonincreasing, and there exists a kernel $l \in L_{1, \text { loc }}\left(\mathbb{R}_{+}\right)$ such that $k * l=1$ in $(0, \infty)$.

In this case we also write $(k, l) \in \mathcal{P C}$. Note that $(k, l) \in \mathcal{P C}$ implies that $l$ is completely positive, cf. [9, Theorem 2.2], in particular $l$ is nonnegative.

Condition $(\mathcal{P C})$ covers most of the relevant integro-differential operators w.r.t. time that appear in physics applications in the context of subdiffusion processes. An important example is given by $(k, l)=\left(g_{1-\alpha}, g_{\alpha}\right)$ with $\alpha \in(0,1)$, where $g_{\beta}$ denotes the standard kernel

$$
g_{\beta}(t)=\frac{t^{\beta-1}}{\Gamma(\beta)}, \quad t>0, \quad \beta>0 .
$$

In this case, the term $\partial_{t}(k * v)$ becomes the Riemann-Liouville fractional derivative $\partial_{t}^{\alpha} v$, and $k * \partial_{t} v={ }^{c} D_{t}^{\alpha} v$, the Caputo fractional derivative (cf. the right-hand side in (3)), of order $\alpha$ (cf. [20]) and (1) is called time-fractional diffusion equation. Condition $(\mathcal{P C})$ also contains the multi-term fractional diffusion case, see Example 4.2 below. Another interesting and important class of examples is given by

$$
k(t)=\int_{0}^{1} g_{\beta}(t) \omega(\beta) d \beta,
$$

where $\omega \in C([0,1])$ is a nonnegative weight function that does not vanish everywhere. In this situation the operator $\partial_{t}(k * \cdot)$ is a so-called operator of distributed order, and (1) is an example of a so-called ultraslow diffusion equation if $\omega(0) \neq 0([21])$. The special case $\omega \equiv 1$ is discussed in Example 4.3 below.

Besides (1), we further investigate the time-fractional diffusion equation with the Laplacian being replaced by a more general elliptic operator in divergence form with rough coefficients, that is, we study

$$
\begin{aligned}
\partial_{t}^{\alpha}\left(u-u_{0}\right)-\operatorname{div}(A(t, x) \nabla u) & =0, \quad t>0, x \in \mathbb{R}^{d}, \\
\left.u\right|_{t=0} & =u_{0}, \quad x \in \mathbb{R}^{d},
\end{aligned}
$$

with a merely measurable, uniformly elliptic coefficient matrix $A$. Thus, our results on weak solutions to (4), (5) do also apply to certain quasilinear equations, take e.g. $A$ of the form $A(t, x)=A_{0}(t, x, u(t, x))$, with some appropriate nonlinear function $A_{0}$.

Applications. Problems of the form (1), in particular the time-fractional diffusion equation, have attracted much interest during the last years, mostly due to their applications in the modeling of anomalous diffusion, see e.g. [21], [23], [26], [38] and the references therein for the physical background. To provide some more specific motivation, let $Z(t, x)$ denote the fundamental solution of (1) satisfying $\left.Z\right|_{t=0}=\delta_{0}$. If $k$ is a kernel of type $(\mathcal{P C})$, then this fundamental solution can be constructed via subordination from the heat kernel and one can show that $Z(t, \cdot)$ is a probability density function (pdf) on $\mathbb{R}^{d}$ for all $t>0$, see the proof of [23, Theorem 3] and Section 2 below. Given a pdf $u_{0}$ on $\mathbb{R}^{d}$ satisfying some appropriate conditions, the solution $u(t, \cdot)$ of the initial-value problem (1), (2) is given by the formula (8) below, and thus is a pdf on $\mathbb{R}^{d}$ for all $t>0$. So in this case, the problem (1), (2) describes the evolution of a pdf on $\mathbb{R}^{d}$.

An important quantity that measures the dispersion of random processes and that describes how fast particles diffuse is the mean square displacement. It can be determined in experiments 
and is defined (in our situation) as

$$
m(t)=\int_{\mathbb{R}^{d}}|x|^{2} Z(t, x) d x, \quad t>0 .
$$

In the case of the classical diffusion equation (i.e. $\alpha=1$ ) $m(t)=c t, t>0$, with some constant $c>0$. In the time-fractional diffusion case (i.e. the first example) one observes that $m(t)=$ $c t^{\alpha}$ (cf. [26]), which shows that the diffusion is slower than in the classical case of Brownian motion. During the recent decades, experimental studies have shown that there is an abundance of processes that have such a power-law mean square displacement, see [4], [26], [27], [38] and the references given therein. An important application is the diffusion on fractals like e.g. some amorphous semiconductors [26], [38]. In our third example, the mean square displacement $m(t)$ behaves like $c \log t$ for $t \rightarrow \infty$ provided that $\omega(0) \neq 0$, see [21]. In this case (1) describes a socalled ultraslow diffusion process. Such processes have been extensively studied recently. They appear, for example, in polymer physics [33], diffusion in disordered media (Sinai diffusion) [36], and in diffusion generated by iterated maps [10]. We would like to point out that in our setting, that is, with a pair of kernels $(k, l) \in \mathcal{P C}$ the mean square displacement is given by

$$
m(t)=2 d(1 * l)(t), \quad t>0,
$$

see Lemma 2.1.

Another important context where equations of the form (1) and nonlinear variants of them appear is the modeling of dynamic processes in materials with memory. They typically arise by some constitutive laws of temporal convolution form (describing the memory of the material) when combined with the usual conservation laws such as balance of energy or balance of momentum. Examples are given by the theory of heat conduction with memory, see e.g. [30] and the references therein as well as [41] (which also contains a quasilinear model), and the diffusion of fluids in porous media with memory, cf. [6], [18].

In view of condition $(\mathcal{P C})$ the problem $(1),(2)$ can be reformulated as an abstract Volterra equation on the positive halfline with a completely positive kernel; this can be seen by convolving the PDE with the kernel $l$. There has been a substantial amount of work on such abstract Volterra and integro-differential equations since the 1970s, in particular on existence and uniqueness, regularity, and long-time behaviour of solutions, see, for instance, [8], [9], [15], [44], and the monograph [30].

One of the main objectives of this paper is to prove sharp estimates for the temporal decay of solutions to (1), (2). We point out that for non-local in space diffusion equations, in particular space-fractional diffusion equations, corresponding results have been obtained recently, see e.g. [3],[5], [7], [17], [39]. Concerning non-local in time diffusion, the case of a bounded domain with homogeneous Dirichlet boundary condition and a kernel of type $\mathcal{P C}$ has been studied recently in [41]. The decay estimates obtained in [41] are optimal and even true in the case of a uniformly elliptic operator in divergence form with rough coefficients, cf. the remarks following formula (15) below. For the time-fractional case with Laplacian we also refer to [25] and [28]. Moreover, decay estimates for the time-fractional diffusion equation in $\mathbb{R}^{d}$ have also gathered interest in the engineering community [24]. However, due to the unexpected critical dimension phenomenon (to be discussed below) some of the subtleties of the equation have been overlooked there.

Our main observation is that the decay behaviour of the non-local in time diffusion models is markedly different from that of the standard caloric functions. We encounter a critical dimension 
phenomenon saying that the decay rate of the bounded domain case is attained for a finite critical dimension and does not anymore improve with increasing dimension. We also prove a series of other decay results, the main results including the following:

1. a quantitative decay rate at which mild solutions of the time-fractional diffusion equation tend in $L_{p}$ to a multiple of the fundamental solution $Z$,

2. optimal $L_{p}$-decay rates via Fourier multiplier methods for the general subdiffusion equation (1) with a kernel $k$ of type $\mathcal{P C}$,

3. an $L_{2}$-decay estimate via energy methods for weak solutions of the time-fractional diffusion equation (4) with rough coefficient matrix $A$.

We will now describe our main results in more detail. Under appropriate conditions on $u_{0}$ the solution to $(1),(2)$ is given by

$$
u(t, x)=\int_{\mathbb{R}^{d}} Z(t, x-y) u_{0}(y) d y, \quad t>0, x \in \mathbb{R}^{d} .
$$

Given $u_{0}$ in some Lebesgue space $L_{q}\left(\mathbb{R}^{d}\right), q \in[1, \infty]$ we want to understand the decay behaviour of $\left|Z(t, \cdot) \star u_{0}\right|_{L_{r}\left(\mathbb{R}^{d}\right)}$ as $t \rightarrow \infty$ for suitable $r \in[1, \infty]$; here $f_{1} \star f_{2}$ denotes the convolution of $f_{1}, f_{2}$ on $\mathbb{R}^{d}$. It turns out that the situation is significantly different from that in the case of the heat equation, where $Z(t, x)=H(t, x)=(4 \pi t)^{-d / 2} \exp \left(-|x|^{2} / 4 t\right)$ is the Gaussian heat kernel.

Time-fractional diffusion. Let us first consider for simplicity the time-fractional diffusion equation (i.e. $\left.k=g_{1-\alpha}, \alpha \in(0,1)\right)$ and the case $r=2$. Given $u_{0} \in L_{2}\left(\mathbb{R}^{d}\right)$ we do not have in general any decay neither for $\left|Z(t, \cdot) \star u_{0}\right|_{L_{2}\left(\mathbb{R}^{d}\right)}$ nor for $\left|H(t, \cdot) \star u_{0}\right|_{L_{2}\left(\mathbb{R}^{d}\right)}$. Now suppose that $u_{0} \in L_{2}\left(\mathbb{R}^{d}\right) \cap L_{1}\left(\mathbb{R}^{d}\right)$. Then it is well-known that $u(t, \cdot):=H(t, \cdot) \star u_{0}$ decays in the $L_{2}$-norm as

$$
|u(t, \cdot)|_{2} \lesssim t^{-\frac{d}{4}}, \quad t>0,
$$

and this estimate is the best one can obtain in general (see e.g. [2]). Here $|v|_{2}:=|v|_{L_{2}\left(\mathbb{R}^{d}\right)}$, and $v(t) \lesssim w(t), t>0$ means that there exists a constant $C>0$ such that $v(t) \leq C w(t), t>0$. In the case of time-fractional diffusion $u(t, \cdot):=Z(t, \cdot) \star u_{0}$ exhibits the decay behaviour

$$
|u(t, \cdot)|_{2} \lesssim t^{-\min \left\{\frac{\alpha d}{4}, \alpha\right\}}, \quad t>0, d \in \mathbb{N} \backslash\{4\}
$$

see Corollary 3.2 below. Whereas for the heat equation the decay rate increases with the dimension $d$, time-fractional diffusion leads to the phenomenon of a critical dimension, which is $d=4$ in this case. Below the critical dimension the rate increases with $d$, the exponent being $\alpha$ times the one from the heat equation, while above the critical dimension the decay rate is the same for all $d$, namely $t^{-\alpha}$. The reason why the decay rate does not increase any further with $d$ lies in the fact that $t^{-\alpha}$ (up to a constant) coincides with the decay rate in the case of a bounded domain and homogeneous Dirichlet boundary condition, cf. the remarks on problem (13) below. This reveals another interesting phenomenon: In the time-fractional case the diffusion is so slow that in higher dimensions ( $d$ above the critical dimension) restriction to a bounded domain and the requirement of a homogeneous Dirichlet boundary condition do not improve the rate of decay. This phenomenon cannot be observed in the classical diffusion case, where we always have exponential (and thus a better) decay in the case of a bounded domain. 
We point out that the estimate (9) is the best one can get in general, see Theorem 4.1 and Example 4.1 below. In the case of the critical dimension $d=4$ we obtain the same decay rate as for $d>4$, however we have to replace the $L_{2}$-norm by the weak $L_{2}$-norm, that is we find that

$$
|u(t, \cdot)|_{2, \infty} \lesssim t^{-\alpha}, \quad t>0
$$

In the more general case where $r \in(1, \infty)$ and $u_{0} \in L_{1}\left(\mathbb{R}^{d}\right) \cap L_{r}\left(\mathbb{R}^{d}\right)$, the critical dimension (which is in general not an integer) is given by

$$
d_{\text {crit }}=\frac{2 r}{r-1}
$$

Assuming $d \geq 3$ we show that $|u(t, \cdot)|_{r} \lesssim t^{-\frac{\alpha d}{2}\left(1-\frac{1}{r}\right)}$, if $d<d_{c r i t}$, and $|u(t, \cdot)|_{r, \infty} \lesssim t^{-\alpha}$, if $d=d_{\text {crit }}$, as well as $|u(t, \cdot)|_{r} \lesssim t^{-\alpha}$, whenever $d>d_{\text {crit }}$. Moreover, in the case $d<3$ we always have a subcritical decay behaviour, see Corollary 3.1 .

The critical dimension phenomenon can be well understood by looking at the asymptotic properties of the fundamental solution $Z$. In the time-fractional case, $Z$ can be represented by Fox-functions and corresponding estimates are known (cf. [12] and Prop. 3.1 below). They show that for $d \geq 2$ the fundamental solution $Z(t, x)$ does not only have a singularity at $t=0$ but also at the origin $x=0$, which is a striking difference to the heat kernel. If, for example, $d \geq 3$ and $t^{-\alpha}|x|^{2} \leq 1$, we have the sharp estimate $Z(t, x) \leq C t^{-\alpha}|x|^{-d+2}$, thus $Z$ is (up to a constant) bounded above by $g_{1-\alpha}(t)$ times the Newtonian potential w.r.t. $x$. This provides some interesting insight into how the fundamental solutions from the Poisson and the heat equation interpolate in case of fractional dynamics.

Relying on the asymptotic bounds for (the time-fractional) $Z$, in Section 3 we first derive estimates for $|Z(t, \cdot)|_{p}$ (for suitable $p \in[1, \infty]$ ), which by Young's inequality then yield bounds for $\left|Z(t, \cdot) \star u_{0}\right|_{r}$. We further look at gradient estimates for $Z(t, \cdot)$ and $Z(t, \cdot) \star u_{0}$, and we show that for integrable initial data $u_{0}$ the asymptotic behaviour of $Z(t, \cdot) \star u_{0}$ as $t \rightarrow \infty$ is described by a multiple of $Z(t, x)$. This is the time-fractional analogue of a well-known result for the heat equation, cf. [31, Prop. 48.6].

The general subdiffusion case and Fourier multiplier methods. Turning to the more general subdiffusion equation (1) with $k$ being a kernel of type $\mathcal{P C}$, we first note that asymptotic bounds for $Z(t, x)$ like those in the time-fractional case do not seem to be known in the general case. However, we mention [21] where the ultraslow-diffusion case was studied. To derive decay estimates for $Z(t, \cdot) \star u_{0}$ we develop a theory which is based on tools from harmonic analysis and a careful estimation of the Fourier symbol $\tilde{Z}(t, \xi)$ of $Z$ w.r.t. the spatial variable.

Taking the Fourier transform w.r.t. $x$ (defined as in (17) below) we see that $\tilde{Z}(t, \xi)$ solves the problem

$$
\partial_{t}(k *[\tilde{Z}-1])+|\xi|^{2} \tilde{Z}=0, \quad t>0, \xi \in \mathbb{R}^{d}, \quad \tilde{Z}(0, \xi)=1, \quad \xi \in \mathbb{R}^{d},
$$

that is, we have

$$
\tilde{Z}(t, \xi)=s\left(t,|\xi|^{2}\right), \quad t \geq 0, \xi \in \mathbb{R}^{d} .
$$

Here the so-called relaxation function $s(t, \mu):=s_{\mu}(t), t \geq 0$, is defined for the parameter $\mu \geq 0$ as the solution of the Volterra integral equation

$$
s_{\mu}(t)+\mu\left(l * s_{\mu}\right)(t)=1, \quad t \geq 0 .
$$


Note that $s_{0} \equiv 1$ and that (10) is equivalent to the integro-differential equation

$$
\frac{d}{d t}\left(k *\left[s_{\mu}-1\right]\right)(t)+\mu s_{\mu}(t)=0, \quad t>0, \quad s_{\mu}(0)=1 .
$$

It is known that the assumption $(k, l) \in \mathcal{P C}$ implies that $s_{\mu}$ is nonnegative, nonincreasing, and that $s_{\mu} \in H_{1, l o c}^{1}\left(\mathbb{R}_{+}\right)$; moreover $\partial_{\mu} s_{\mu}(t) \leq 0$, see e.g. Prüss [30]. Furthermore, it is known (see e.g. [41]) that for any $\mu \geq 0$ there holds

$$
\frac{1}{1+\mu k(t)^{-1}} \leq s_{\mu}(t) \leq \frac{1}{1+\mu(1 * l)(t)}, \quad \text { a.a. } t>0,
$$

which also shows that

$$
\left[1-s_{\mu}(t)\right] k(t) \leq \mu s_{\mu}(t) \leq\left[1-s_{\mu}(t)\right] \frac{1}{(1 * l)(t)}, \quad \text { a.a. } t>0 .
$$

This implies that for any fixed $\mu>0, s_{\mu}(t)$ cannot decay faster than the kernel $k(t)$, and $s_{\mu}(t)$ decays at least like $(1 * l)(t)^{-1}$. Note that $\lim _{t \rightarrow \infty} s_{\mu}(t)=0$ if and only if $l \notin L_{1}\left(\mathbb{R}_{+}\right)$, see e.g. [41, Lemma 6.1].

By means of (11) and Plancherel's theorem we are able to prove the following $L_{2}$-decay estimate. Suppose $u_{0} \in L_{1}\left(\mathbb{R}^{d}\right) \cap L_{2}\left(\mathbb{R}^{d}\right)$, then

$$
\left|Z(t, \cdot) \star u_{0}\right|_{2} \lesssim[(1 * l)(t)]^{-\min \left\{1, \frac{d}{4}\right\}}, \quad t>0, d \in \mathbb{N} \backslash\{4\},
$$

see Theorem 4.2. We also show that this estimate gives the optimal rate of decay provided $(1 * l)(t)^{-1} \leq C k(t)$ for $t \geq T$, where $C, T$ are some positive constants. This applies in particular to the time-fractional diffusion equation, where $k(t)$ and $(1 * l)(t)^{-1}=g_{1+\alpha}(t)^{-1}$ decay like $c t^{-\alpha}$, and to the ultraslow diffusion equation, where $k(t)$ and $(1 * l)(t)^{-1}$ decay like $c(\log t)^{-1}$. (12) also reveals that the phenomenon of critical dimension extends to the general case of a kernel $k$ of type $\mathcal{P C}$.

In order to obtain sharp $L_{r}$-decay estimates and to treat the critical dimension case we go a step further and derive bounds for the partial derivatives of arbitrary order of the functions $\xi \rightarrow|\xi|^{2 \delta} \tilde{Z}(t, \xi)$, where $t>0$ is fixed and $\delta \in(0,1]$ is a parameter. These bounds are uniform w.r.t. time and allow us to apply Mihlin's multiplier theorem. This leads to the desired $L_{r^{-}}$ (and by interpolation also to the $L_{r, \infty^{-}}$) decay estimates, which generalize those from the timefractional case. Here in our analysis we make use of another important property of the relaxation function $s(t, \mu)$ which is the complete monotonicity w.r.t. to the parameter $\mu$ for all $t \geq 0$, see Section 5 below. This property has already been known, e.g. it follows from results in [30, Section 4]. In the present paper we provide a new proof of this property, which is rather short and relies on a comparison principle for a certain type of integro-differential equation.

By switching the kernels from the ultraslow diffusion case one obtains an interesting example of a pair $(k, l) \in \mathcal{P C}$ where $k(t)$ decays like $t^{-1}$ and $(1 * l)(t)^{-1}$ like $c t^{-1} \log t$. Here the upper bound in (11) does not lead to the optimal decay. However, it has been shown in [41] that $s_{\mu}(t) \leq \frac{C}{1+\mu t}$ for all $t, \mu \geq 0$, and thus a simple modification of our original proof yields the optimal $L_{2}$-estimate

$$
\begin{aligned}
|u(t, \cdot)|_{2} & \lesssim t^{-\min \left\{1, \frac{d}{4}\right\}}, \quad t>0, d \in \mathbb{N} \backslash\{4\}, \\
|u(t, \cdot)|_{2, \infty} & \lesssim t^{-1}, \quad t>0, d=4,
\end{aligned}
$$


provided $u_{0} \in L_{1}\left(\mathbb{R}^{d}\right) \cap L_{2}\left(\mathbb{R}^{d}\right)$, cf. Example 4.4. This is a remarkable result as for $d \leq 3$ the decay rate is the same as for the heat equation!

Known results in the bounded domain case. It is instructive to compare our decay results with what is known in the case of a bounded domain and a homogeneous Dirichlet condition. Let $\Omega \subset \mathbb{R}^{d}$ be a bounded domain, $u_{0} \in L_{2}(\Omega)$, and assume again that $k$ is of type $\mathcal{P C}$. We consider the problem

$$
\begin{aligned}
\partial_{t}\left(k *\left[u-u_{0}\right]\right)-\Delta u & =0, \quad t>0, x \in \Omega, \\
\left.u\right|_{\partial \Omega} & =0, \quad t>0, x \in \partial \Omega, \\
\left.u\right|_{t=0} & =u_{0}, \quad x \in \partial \Omega .
\end{aligned}
$$

Let $\left\{\phi_{n}\right\}_{n=1}^{\infty} \subset \stackrel{\circ}{H}_{2}^{1}(\Omega):=\overline{C_{0}^{\infty}(\Omega)} H_{2}^{1}(\Omega)$ be an orthonormal basis of $L_{2}(\Omega)$ consisting of eigenfunctions of the negative Dirichlet Laplacian with eigenvalues $\lambda_{n}>0, n \in \mathbb{N}$, and denote by $\lambda_{1}$ the smallest such eigenvalue. Then the solution $u$ of (13) can be represented via Fourier series as

$$
u(t, x)=\sum_{n=1}^{\infty} s_{\lambda_{n}}(t)\left(u_{0} \mid \phi_{n}\right) \phi_{n}(x), \quad t \geq 0, x \in \Omega,
$$

where $(\cdot \mid \cdot)$ stands for the standard inner product in $L_{2}(\Omega)$, cf. [41, Section 1], the special case $k=g_{1-\alpha}$ can be also found in [28, Theorem 4.1]. By Parseval's identity and since $\partial_{\mu} s_{\mu} \leq 0$, it follows from (14) that

$$
\begin{aligned}
|u(t, \cdot)|_{L_{2}(\Omega)}^{2} & =\sum_{n=1}^{\infty} s_{\lambda_{n}}^{2}(t)\left|\left(u_{0} \mid \phi_{n}\right)\right|^{2} \\
& \leq s_{\lambda_{1}}^{2}(t) \sum_{n=1}^{\infty}\left|\left(u_{0} \mid \phi_{n}\right)\right|^{2} \\
& =s_{\lambda_{1}}^{2}(t)\left|u_{0}\right|_{L_{2}(\Omega)}^{2}
\end{aligned}
$$

and thus

$$
|u(t, \cdot)|_{L_{2}(\Omega)} \leq s_{\lambda_{1}}(t)\left|u_{0}\right|_{L_{2}(\Omega)}, \quad t \geq 0,
$$

cf. [41]. This decay estimate is optimal as the example $u_{0}=\phi_{1}$ with solution $u(t, x)=s_{\lambda_{1}}(t) \phi_{1}(x)$ shows. By means of energy methods (15) can be generalized to problems with a uniformly elliptic operator in divergence form with rough coefficients, cf. [41, Corollary 1.1]. (15) can be further extended to $r \in(1, \infty)$, in fact assuming $u_{0} \in L_{r}(\Omega)$ and setting $\rho(r):=4(r-1) / r^{2}$ we have

$$
|u(t, \cdot)|_{r} \leq s_{\lambda_{1} \rho(r)}(t)\left|u_{0}\right|_{r}, \quad t>0,
$$

see [41, Remark 5.1]. We see that the relaxation function $s_{\mu}(t)$ (with some fixed $\mu>0$ ) determines the rate of decay as $t \rightarrow \infty$. For example, if $k=g_{1-\alpha}$ with $\alpha \in(0,1)$ it follows from (11) that $s_{\mu}(t)$ decays like $c t^{-\alpha}$. This justifies among others earlier remarks on (9) concerning the case $d>4$.

Energy methods for weak solutions. A further goal of this paper is to prove decay estimates for the time-fractional diffusion equation (4), (5), where instead of the Laplacian a more general elliptic operator in divergence form with rough coefficients is considered. We 
assume $\alpha \in(0,1), u_{0} \in L_{1}\left(\mathbb{R}^{d}\right) \cap L_{2}\left(\mathbb{R}^{d}\right), A \in L_{\infty, l o c}\left([0, \infty) \times \mathbb{R}^{d} ; \mathbb{R}^{d \times d}\right)$, and that $A$ satisfies a uniform parabolicity condition. We prove that if $u$ is a suitably defined weak solution of (4), (5) satisfying appropriate integrability conditions at $x=\infty$, there holds for all $d \in \mathbb{N}$

$$
|u(t)|_{2} \lesssim t^{-\frac{\alpha d}{d+4}}, \quad t>0
$$

see Theorem 6.1. The basic idea of the proof is to show that for some constant $\mu>0$

$$
\partial_{t}^{\alpha}\left(|u|_{2}-\left|u_{0}\right|_{2}\right)(t)+\mu|u(t)|_{2}^{1+\frac{4}{d}} \leq 0, \quad t>0
$$

in the weak sense. This can be achieved by means of Nash's inequality and the so-called $L_{p^{-}}$ norm inequality for operators of the form $\partial_{t}(k * \cdot)$, which has been established recently in [41], cf. Lemma 6.2 below. Note that the decay rate in (16) is less than the one we find in the case of the Laplacian (cf. (9)). This phenomenon of a smaller decay rate in the weak setting with rough coefficients does not occur in the case $\alpha=1$, where the same strategy of proof leads to the optimal decay rate $t^{-d / 4}$. It is an interesting open problem whether (16) provides the optimal decay rate in the variational setting.

The paper is organized as follows. In Section 2 we provide some background on the fundamental solution $Z$, including their construction via subordination. Section 3 is devoted to the timefractional diffusion case. We study the decay properties of $Z$ and $Z(t, \cdot) \star u_{0}$, respectively. Sections 4 and 5 deal with the general case. We first prove $L_{2}$-decay estimates for $Z(t, \cdot) \star u_{0}$ using Plancherel's theorem and discuss some specific examples of pairs $(k, l) \in \mathcal{P C}$ (Section 4 ). Then we establish $L_{r}$-estimates and look at the critical dimension case (Section 5 ). Finally, in Section 6 we discuss decay estimates in the variational setting.

\section{The fundamental solution $Z$}

Suppose $(k, l) \in \mathcal{P C}$. We will describe how the fundamental solution $Z$ to the subdiffusion problem (1), (2) can be constructed from the Gaussian heat kernel $H$ by means of the subordination principle for abstract Volterra equations with completely positive kernels, cf. Prüss [30, Chapter 4]. See also the proof of [23, Theorem 3]. We will also show that $Z(t, \cdot)$ is a probability density function on $\mathbb{R}^{d}$ for all $t>0$.

By definition, the fundamental solution $Z(t, x)$ to the subdiffusion problem (1), (2) is a distributional solution of

$$
\partial_{t}\left(k *\left[Z-Z_{0}\right]\right)-\Delta Z=0, \quad t>0, x \in \mathbb{R}^{d},\left.\quad Z\right|_{t=0}=Z_{0}:=\delta_{0}, x \in \mathbb{R}^{d},
$$

where $\delta_{0}$ stands for the Dirac delta distribution. Throughout this paper the Fourier transform of $v \in \mathcal{S}\left(\mathbb{R}^{d}\right)$ is defined by

$$
\tilde{v}(\xi)=\int_{\mathbb{R}^{d}} e^{-i x \cdot \xi} v(x) d x,
$$

extended as usual to $\mathcal{S}^{\prime}\left(\mathbb{R}^{d}\right)$. Taking the Fourier transform w.r.t. $x$ in the problem for $Z$ we obtain (cf. Section 1)

$$
\tilde{Z}(t, \xi)=s\left(t,|\xi|^{2}\right), \quad t \geq 0, \xi \in \mathbb{R}^{d},
$$


where the relaxation function $s(t, \mu)$ is defined via the Volterra equation (10). In what follows we will construct a function $Z$ that enjoys the latter property.

Note first that $(k, l) \in \mathcal{P C}$ implies that the kernel $l$ is completely positive, see [9] and [30]. Denoting by $\hat{f}$ the Laplace transform of $f: \mathbb{R}_{+} \rightarrow \mathbb{R}$, this in turn implies that $\varphi(\lambda)=\lambda \hat{k}(\lambda), \lambda>$ 0 , is a Bernstein function and that for every $\tau \geq 0$, the function $\psi_{\tau}(\lambda)=\exp (-\tau \varphi(\lambda)), \lambda>0$, is completely monotone, by [30, Proposition 4.5]. Further, $\psi_{\tau}(\lambda)$ is bounded by $e^{-\tau \varphi(0+)}$. Note that $\varphi(\lambda)=1 / \hat{l}(\lambda)$, and thus $\varphi(0+)=1 /|l|_{L_{1}\left(\mathbb{R}_{+}\right)}$, which is 0 if and only if $l \notin L_{1}\left(\mathbb{R}_{+}\right)$. By Bernstein's theorem (see e.g. [30, Section 4.1] or [34, Theorem 1.4]) there exist unique nondecreasing functions $w(\cdot, \tau) \in B V\left(\mathbb{R}_{+}\right)$normalized by $w(0, \tau)=0$ and left-continuity such that

$$
\hat{w}(\lambda, \tau)=\int_{0}^{\infty} e^{-\lambda \sigma} w(\sigma, \tau) d \sigma=\frac{\psi_{\tau}(\lambda)}{\lambda}, \quad \lambda>0 .
$$

The function $w(t, \tau)$ is the so-called propagation function associated with the completely positive kernel $l$, cf. [30, Section 4.5]. Some important properties of $w(t, \tau)$ can be found in [30, Proposition 4.9]. Among others, $w(\cdot, \cdot)$ is Borel measurable on $\mathbb{R}_{+} \times \mathbb{R}_{+}, w(t, \cdot)$ is nonincreasing and rightcontinuous on $\mathbb{R}_{+}$, and $w(t, 0)=w(t, 0+)=1$ as well as $w(t, \infty)=0$ for all $t>0$. Moreover, the relaxation function $s(t, \mu)$ is represented by

$$
s(t, \mu)=-\int_{0}^{\infty} e^{-\mu \tau} d_{\tau} w(t, \tau), \quad t>0, \mu \geq 0,
$$

in particular $-\int_{0}^{\infty} d_{\tau} w(t, \tau)=1$

We now set

$$
Z(t, x)=-\int_{0}^{\infty} H(\tau, x) d_{\tau} w(t, \tau), \quad t>0, x \in \mathbb{R}^{d} .
$$

Then $Z(t, x)$ is nonnegative, and $|Z(t, \cdot)|_{1}=1$ for all $t>0$, since $H$ enjoys these properties. Taking the Fourier transform w.r.t. $x$ and using (18) we obtain

$$
\tilde{Z}(t, \xi)=-\int_{0}^{\infty} \tilde{H}(t, \xi) d_{\tau} w(t, \tau)=-\int_{0}^{\infty} e^{-\tau|\xi|^{2}} d_{\tau} w(t, \tau)=s\left(t,|\xi|^{2}\right) .
$$

Thus $Z$ is the desired fundamental solution to (1).

The following lemma provides a formula for the mean square displacement. The basic idea of the proof can be found already in [26, p. 19] (time-fractional case with $d=1$ ) and [21, p. 268] (ultraslow diffusion with arbitrary $d$ ).

Lemma 2.1 Let $(k, l) \in \mathcal{P C}$ and $Z$ be the fundamental solution to the diffusion problem (1), (2). Let $m(t)$ be defined as in (6). Then

$$
m(t)=2 d(1 * l)(t), \quad t>0 .
$$


Proof. For the Laplace transform of $m$ we have

$$
\begin{aligned}
\hat{m}(\lambda) & =\int_{0}^{\infty} \int_{\mathbb{R}^{d}}|x|^{2} e^{-\lambda t} Z(t, x) d x d t \\
& =\left.\int_{0}^{\infty} \int_{\mathbb{R}^{d}}\left(-\Delta_{\xi} e^{-i x \cdot \xi}\right)\right|_{\xi=0} e^{-\lambda t} Z(t, x) d x d t \\
& =\left.\left(-\Delta_{\xi} \int_{0}^{\infty} \int_{\mathbb{R}^{d}} e^{-i x \cdot \xi} e^{-\lambda t} Z(t, x) d x d t\right)\right|_{\xi=0} \\
& =\left.\left(-\Delta_{\xi} \hat{\tilde{Z}}(\lambda, \xi)\right)\right|_{\xi=0} \\
& =\left.\left(-\Delta_{\xi} \hat{s}\left(\lambda,|\xi|^{2}\right)\right)\right|_{\xi=0} \\
& =\left.\left(-\Delta_{\xi}\left[\frac{1}{\lambda\left(1+|\xi|^{2} \hat{l}(\lambda)\right)}\right]\right)\right|_{\xi=0} \\
& =2 d \frac{\hat{l}(\lambda)}{\lambda},
\end{aligned}
$$

and thus the claim follows by inversion of the Laplace transform.

Let us illustrate Lemma 2.1 by looking at the time-fractional diffusion case, where $k=g_{1-\alpha}$ and $l=g_{\alpha}$ with some $\alpha \in(0,1)$. The formula for $m(t)$ gives

$$
m(t)=2 d\left(1 * g_{\alpha}\right)(t)=2 d g_{1+\alpha}(t)=\frac{2 d}{\Gamma(1+\alpha)} t^{\alpha}, \quad t>0,
$$

which is in accordance with our remarks in the paragraph following (6) in Section 1.

\section{Time-fractional diffusion}

In this section we study the subdiffusion problem (1), (2) in the important special case $k=g_{1-\alpha}$ with $\alpha \in(0,1)$. That is, we consider the problem

$$
\begin{aligned}
\partial_{t}^{\alpha}\left(u-u_{0}\right)-\Delta u & =0, \quad t>0, x \in \mathbb{R}^{d}, \\
\left.u\right|_{t=0} & =u_{0}, \quad x \in \mathbb{R}^{d} .
\end{aligned}
$$

Under appropriate conditions on $u_{0}$ the solution of (19), (20) can be represented as

$$
u(t, x)=\int_{\mathbb{R}^{d}} Z(t, x-y) u_{0}(y) d y,
$$

where $Z$ is the fundamental solution corresponding to $(19),(20)$, see [12]. It is known (see e.g. $[22],[35])$ that

$$
Z(t, x)=\pi^{-\frac{d}{2}} t^{\alpha-1}|x|^{-d} H_{12}^{20}\left(\left.\frac{1}{4}|x|^{2} t^{-\alpha}\right|_{(d / 2,1),(1,1)} ^{(\alpha, \alpha)}\right), \quad t>0, x \in \mathbb{R}^{d} \backslash\{0\},
$$

where $H$ denotes the Fox $H$-function ([19], [20]). As the $H$-function is a rather complicated object, this representation of $Z$ is not so useful for deriving estimates for $Z$ directly. However, 
using the analytic and asymptotic properties of $H$, one can obtain the subsequent sharp estimates, which can be found in [12], see also [22].

Proposition 3.1 Set $R=t^{-\alpha}|x|^{2}$. Then

$$
\begin{aligned}
Z(t, x) & \leq C t^{-\frac{\alpha d}{2}} \exp \left(-\sigma R^{\frac{1}{2-\alpha}}\right), \quad \text { if } R \geq 1, \\
Z(t, x) & \leq C t^{-\alpha}|x|^{-d+2}, \quad \text { if } R \leq 1 \text { and } d \geq 3, \\
Z(t, x) & \leq C t^{-\alpha}(|\log R|+1), \quad \text { if } R \leq 1 \text { and } d=2, \\
Z(t, x) & \leq C t^{-\frac{\alpha}{2}}, \quad \text { if } R \leq 1 \text { and } d=1, \\
|\nabla Z(t, x)| & \leq C t^{-\frac{\alpha(d+1)}{2}} \exp \left(-\sigma R^{\frac{1}{2-\alpha}}\right), \quad \text { if } R \geq 1, \\
|\nabla Z(t, x)| & \leq C t^{-\alpha}|x|^{-d+1}, \quad \text { if } R \leq 1 \text { and } d \geq 2, \\
|\nabla Z(t, x)| & \leq C t^{-\alpha}, \quad \text { if } R \leq 1 \text { and } d=1 .
\end{aligned}
$$

Here $C=C(\alpha, d)$ is a positive constant which may differ from line to line.

We point out that for $d \geq 2$ the fundamental solution $Z(t, x)$ has a singularity at the origin $x=0$, which is a fundamental difference to the heat kernel and which leads to a restriction concerning $p$-integrability on $\mathbb{R}^{d}$.

\section{1 $L_{p}\left(\mathbb{R}^{d}\right)$-estimates for $Z$ and the solution}

Proposition 3.1 allows us to estimate the $L_{p}\left(\mathbb{R}^{d}\right)$-norm of $Z(t, \cdot)$ for $t>0$. We decompose the corresponding integral as follows.

$$
|Z(t)|_{p}^{p} \leq \int_{\{R \geq 1\}} Z(t, x)^{p} d x+\int_{\{R \leq 1\}} Z(t, x)^{p} d x .
$$

For all dimensions $d$ and $1<p<\infty$, we have in view of (22)

$$
\begin{aligned}
\int_{\{R \geq 1\}} Z(t, x)^{p} d x & \lesssim \int_{\{R \geq 1\}} t^{-\frac{\alpha d p}{2}} \exp \left(-\sigma p R^{\frac{1}{2-\alpha}}\right) d x \\
& \lesssim \int_{t^{\frac{\alpha}{2}}}^{\infty} t^{-\frac{\alpha d p}{2}} \exp \left(-\sigma p\left(r^{2} t^{-\alpha}\right)^{\frac{1}{2-\alpha}}\right) r^{d-1} d r \\
& \lesssim t^{-\frac{\alpha d p}{2}+\frac{\alpha d}{2}} \int_{1}^{\infty} \exp \left(-\sigma p s^{\frac{2}{2-\alpha}}\right) s^{d-1} d s
\end{aligned}
$$

and thus

$$
\left(\int_{\{R \geq 1\}} Z(t, x)^{p} d x\right)^{\frac{1}{p}} \lesssim t^{-\frac{\alpha d}{2}\left(1-\frac{1}{p}\right)}, \quad t>0, \quad \text { for all } 1<p<\infty .
$$

We come now to the estimate for the integral where $R \leq 1$. In case $d=1$ we have in view of (25) for all $1<p<\infty$

$$
\int_{\{R \leq 1\}} Z(t, x)^{p} d x \lesssim \int_{\{R \leq 1\}} t^{-\frac{\alpha p}{2}} d x \lesssim \int_{0}^{t^{\frac{\alpha}{2}}} t^{-\frac{\alpha p}{2}} d x \lesssim t^{-\frac{\alpha p}{2}+\frac{\alpha}{2}}
$$


If $d=2$ we may estimate as follows, employing (24).

$$
\begin{aligned}
\int_{\{R \leq 1\}} Z(t, x)^{p} d x & \lesssim \int_{\{R \leq 1\}} t^{-\alpha p}(|\log R|+1)^{p} d x \\
& \lesssim \int_{0}^{t^{\frac{\alpha}{2}}} t^{-\alpha p}\left(\left|\log \left(r^{2} t^{-\alpha}\right)\right|+1\right)^{p} r d r \\
& \lesssim \int_{0}^{1} t^{-\alpha p+\alpha}\left(\left|\log \left(s^{2}\right)\right|+1\right)^{p} s d s \lesssim t^{-\alpha p+\alpha},
\end{aligned}
$$

for all $1<p<\infty$. For $d \geq 3$ we use (23) to obtain

$$
\begin{aligned}
\int_{\{R \leq 1\}} Z(t, x)^{p} d x & \lesssim \int_{\{R \leq 1\}} t^{-\alpha p}|x|^{(-d+2) p} d x \lesssim \int_{0}^{t^{\frac{\alpha}{2}}} t^{-\alpha p} r^{(-d+2) p} r^{d-1} d r \\
& \lesssim t^{-\alpha p+\frac{\alpha}{2}(d+[2-d] p)} \int_{0}^{1} s^{(-d+2) p} s^{d-1} d s \lesssim t^{-\frac{\alpha d}{2}(p-1)}
\end{aligned}
$$

whenever the last integral is finite, that is, whenever

$$
p<\frac{d}{d-2}=: \kappa(d) .
$$

Setting $\kappa(1)=\kappa(2)=\infty$ and combining the previous estimates we see that

$$
\left(\int_{\{R \leq 1\}} Z(t, x)^{p} d x\right)^{\frac{1}{p}} \lesssim t^{-\frac{\alpha d}{2}\left(1-\frac{1}{p}\right)}, \quad t>0, \quad \text { for all } 1<p<\kappa(d) .
$$

Observe also that $Z(t) \in L_{\infty}(\mathbb{R}), t>0$, provided that $d=1$, and we have the estimate $|Z(t)|_{\infty} \lesssim t^{-\alpha / 2}$.

Summarizing we have proved

Theorem 3.1 Let $d \in \mathbb{N}$ and $\kappa(d)$ be as above. Then $Z(t)$ belongs to $L_{p}\left(\mathbb{R}^{d}\right)$ for all $t>0$, provided that $1 \leq p<\kappa(d)$, and there holds

$$
|Z(t)|_{p} \lesssim t^{-\frac{\alpha d}{2}\left(1-\frac{1}{p}\right)}, \quad t>0 .
$$

(27) remains true for $d=1$ and $p=\kappa(1)=\infty$.

Remark 3.1 $Z(t)$ fails to belong to $L_{p}\left(\mathbb{R}^{d}\right)$ for $d \geq 4$ and $p \geq \kappa(d)=d /(d-2)$. In fact, taking the Fourier transform w.r.t. the spatial variables we see that the Fourier transform of $Z(t, x)$ solves the fractional differential equation

$$
\partial_{t}^{\alpha}(\tilde{Z}-1)+|\xi|^{2} \tilde{Z}=0, \quad t>0, \quad \tilde{Z}(0)=1 .
$$

Thus

$$
\tilde{Z}(t, \xi)=s\left(t,|\xi|^{2}\right)=E_{\alpha}\left(-|\xi|^{2} t^{\alpha}\right),
$$


where $E_{\alpha}(z)$ denotes the Mittag-Leffler function, defined by

$$
E_{\alpha}(z)=\sum_{j=0}^{\infty} \frac{z^{j}}{\Gamma(\alpha j+1)}, \quad z \in \mathbb{C} .
$$

Employing the bounds from (11), a short computation shows that the Mittag-Leffler function satisfies the estimate

$$
\frac{1}{1+\Gamma(1-\alpha) x} \leq E_{\alpha}(-x) \leq \frac{1}{1+\frac{x}{\Gamma(1+\alpha)}}, \quad x \geq 0,
$$

see also [41, Example 6.1]. An upper bound of the form $E_{\alpha}(-x) \leq C(\alpha) /(1+x), x \geq 0$, can also be found in [25].

Suppose now that $Z(t)$ belongs to $L_{p}\left(\mathbb{R}^{d}\right)$ with some $p \leq 2$. Then the Hausdorff-Young inequality implies that

$$
|\tilde{Z}(t)|_{p^{\prime}} \lesssim|Z(t)|_{p}<\infty,
$$

where $p^{\prime}$ is the conjugate exponent of $p$. From this and the representation of $\tilde{Z}$ as well as (28) it follows that

$$
\int_{\mathbb{R}^{d}} \frac{d \xi}{\left(1+|\xi|^{2} t^{\alpha}\right)^{p^{\prime}}}<\infty
$$

which in turn implies (by changing to polar coordinates) that

$$
\int_{0}^{\infty} \frac{r^{d-1}}{\left(1+r^{2}\right)^{p^{\prime}}} d r<\infty
$$

Hence $2 p^{\prime}-(d-1)>1$, which is equivalent to $p<d /(d-2)$.

We next examine the critical case $p=\frac{d}{d-2}$ for $d \geq 3$. We know that $Z(t)$ does not belong to $L_{p}\left(\mathbb{R}^{d}\right)$. However, it lies in the corresponding weak $L_{p}$-space as the following theorem shows. This observation will be crucial, among others, to obtain optimal decay estimates for $|u(t)|_{2}$ for $d \geq 4$.

Theorem 3.2 Let $d \geq 3$ and $t>0$. Then $Z(t)$ belongs to the space $L_{\frac{d}{d-2}, \infty}\left(\mathbb{R}^{d}\right)$, and there holds the estimate

$$
|Z(t)|_{\frac{d}{d-2}, \infty} \lesssim t^{-\alpha}, \quad t>0 .
$$

Proof. Set $p=\frac{d}{d-2}$. We need to estimate

$$
|Z(t)|_{p, \infty}=\sup \left\{\lambda d_{Z(t)}(\lambda)^{\frac{1}{p}}: \lambda>0\right\},
$$

where

$$
d_{Z(t)}(\lambda)=\left|\left\{x \in \mathbb{R}^{d}: Z(t, x)>\lambda\right\}\right|
$$

denotes the distribution function of $Z(t)$. Using again the similarity variable $R=t^{-\alpha}|x|^{2}$ we have

$$
|Z(t)|_{p, \infty} \leq 2\left(\left|Z(t) \chi_{\{R \leq 1\}}(t)\right|_{p, \infty}+\left|Z(t) \chi_{\{R \geq 1\}}(t)\right|_{p, \infty}\right) .
$$


Employing (26), we find that

$$
\left|Z(t) \chi_{\{R \geq 1\}}(t)\right|_{p, \infty} \leq\left|Z(t) \chi_{\{R \geq 1\}}(t)\right|_{p} \leq C t^{-\frac{\alpha d}{2}\left(1-\frac{1}{p}\right)}=C t^{-\alpha} .
$$

For the term with $R \leq 1$ we use (23) to estimate as follows.

$$
\begin{aligned}
d_{Z(t) \chi_{\{R \leq 1\}}(t)}(\lambda) & =\mid\left\{x \in \mathbb{R}^{d}: Z(t, x)>\lambda \text { and } R \leq 1\right\} \mid \\
& \leq\left|\left\{x \in \mathbb{R}^{d}: \lambda<C t^{-\alpha}|x|^{-d+2}\right\}\right| \\
& =\left|\left\{x \in \mathbb{R}^{d}:|x|<\left(C t^{-\alpha} \lambda^{-1}\right)^{\frac{1}{d-2}}\right\}\right| \\
& \leq C_{1}\left(t^{-\alpha} \lambda^{-1}\right)^{\frac{d}{d-2}} .
\end{aligned}
$$

This shows that

$$
d_{Z(t) \chi_{\{R \leq 1\}}(t)}(\lambda)^{1 / p} \leq C_{1}^{1 / p} t^{-\alpha} \lambda^{-1}
$$

and thus

$$
\left|Z(t) \chi_{\{R \leq 1\}}(t)\right|_{p, \infty} \lesssim t^{-\alpha}
$$

This proves the theorem.

We come now to decay estimates for the $L_{r}\left(\mathbb{R}^{d}\right)$-norm of the solution $u$ of (19), (20) given by formula (21).

Theorem 3.3 (i) Let $d \in \mathbb{N}, 1 \leq p<\kappa(d), 1 \leq q, r \leq \infty, 1+\frac{1}{r}=\frac{1}{p}+\frac{1}{q}$, and $u_{0} \in L_{q}\left(\mathbb{R}^{d}\right)$. Then for $u(t)=Z(t) \star u_{0}$ we have

$$
|u(t)|_{r} \lesssim t^{-\frac{\alpha d}{2}\left(1-\frac{1}{p}\right)}, \quad t>0 .
$$

This estimate remains true for $d=q=1$ and $p=r=\infty$.

(ii) Let $d \geq 3,1<q, r<\infty, \frac{1}{r}+\frac{2}{d}=\frac{1}{q}$, and $u_{0} \in L_{q}\left(\mathbb{R}^{d}\right)$. Then for $u(t)=Z(t) \star u_{0}$ we have

$$
|u(t)|_{r} \lesssim t^{-\alpha}, \quad t>0 .
$$

(iii) Let $d \geq 3$ and $u_{0} \in L_{1}\left(\mathbb{R}^{d}\right)$. Then for $u(t)=Z(t) \star u_{0}$ we have

$$
|u(t)|_{\frac{d}{d-2}, \infty} \lesssim t^{-\alpha}, \quad t>0 .
$$

Proof. (i) By Young's inequality and Theorem 3.1 we have

$$
|u(t)|_{r} \leq|Z(t)|_{p}\left|u_{0}\right|_{q} \lesssim t^{-\frac{\alpha d}{2}\left(1-\frac{1}{p}\right)}, \quad t>0 .
$$

(ii) We apply Young's inequality for weak type spaces (cf. [14, Theorem 1.4.24]) with $p=$ $\kappa(d)=\frac{d}{d-2}$ and invoke Theorem 3.2. This yields

$$
|u(t)|_{r} \leq C(p, q, r)|Z(t)|_{p, \infty}\left|u_{0}\right|_{q} \lesssim t^{-\alpha}, \quad t>0 .
$$

(iii) The assertion follows from Theorem 3.2 and Young's inequality for weak type spaces (see [14, Theorem 1.2.13]). 
Corollary 3.1 Let $1<r<\infty, d \in \mathbb{N}, u_{0} \in L_{1}\left(\mathbb{R}^{d}\right) \cap L_{r}\left(\mathbb{R}^{d}\right)$, and $u(t)=Z(t) \star u_{0}$. Then

$$
\begin{aligned}
|u(t)|_{r} & \lesssim t^{-\frac{\alpha d}{2}\left(1-\frac{1}{r}\right)}, \quad t>0, \quad \text { if } d<3, \\
|u(t)|_{r} & \lesssim t^{-\frac{\alpha d}{2}\left(1-\frac{1}{r}\right)}, \quad t>0, \quad \text { if } d \geq 3 \text { and } d<\frac{2 r}{r-1}, \\
|u(t)|_{r, \infty} & \lesssim t^{-\alpha}, \quad t>0, \quad \text { if } d \geq 3 \text { and } d=\frac{2 r}{r-1}, \\
|u(t)|_{r} & \lesssim t^{-\alpha}, \quad t>0, \quad \text { if } d \geq 3 \text { and } d>\frac{2 r}{r-1} .
\end{aligned}
$$

Proof. If $d<3$ or we have both $d \geq 3$ and $d<\frac{2 r}{r-1}$ then $r<\kappa(d)$ and the first two estimates follow from Theorem 3.3 (i) with $q=1$. The third estimate is a consequence of Theorem 3.3 (iii), since $d=\frac{2 r}{r-1}$ is equivalent to $r=\kappa(d)$ whenever $d \geq 3$. To show the last estimate, observe first that the assumptions on $r$ and $d$ imply that there is $q \in(1, r)$ such that $\frac{1}{r}+\frac{2}{d}=\frac{1}{q}$. The assertion then follows from Theorem 3.3 (ii).

Specializing Corollary 3.1 to $r=2$ we obtain

Corollary 3.2 Let $d \in \mathbb{N}$ and $u_{0} \in L_{1}\left(\mathbb{R}^{d}\right) \cap L_{2}\left(\mathbb{R}^{d}\right)$ and $u(t)=Z(t) \star u_{0}$. Then

$$
\begin{aligned}
|u(t)|_{2} & \lesssim t^{-\min \left\{\frac{\alpha d}{4}, \alpha\right\}}, \quad t>0, d \in \mathbb{N} \backslash\{4\}, \\
|u(t)|_{2, \infty} & \lesssim t^{-\alpha}, \quad t>0, d=4 .
\end{aligned}
$$

Corollary 3.1 exhibits the critical dimension phenomenon discussed already in Section 1. The critical dimension (which is in general not an integer) is here given by $d_{c r i t}=2 r /(r-1)$. As long as the dimension $d$ is below $d_{\text {crit }}$ (and at least 3 ) the decay rate increases with the dimension, whereas for any $d>d_{\text {crit }}$ the decay rate is the same, namely $t^{-\alpha}$, which coincides with the decay rate for the corresponding problem on a bounded domain with homogeneous Dirichlet boundary condition.

\subsection{Gradient estimates}

We turn now to $L_{p}$-estimates for the spatial gradient of $Z(t, x)$. For $d \in \mathbb{N}$ we set $\kappa_{1}(d):=\frac{d}{d-1}$ for $d \geq 2$ and $\kappa(1):=\infty$

Theorem 3.4 (i) Let $d \in \mathbb{N}$. Then $\nabla Z(t)$ belongs to $L_{p}\left(\mathbb{R}^{d} ; \mathbb{R}^{d}\right)$ for all $t>0$, provided that $1 \leq p<\kappa_{1}(d)$, and there holds

$$
|\nabla Z(t)|_{p} \lesssim t^{-\frac{\alpha}{2}-\frac{\alpha d}{2}\left(1-\frac{1}{p}\right)}, \quad t>0 .
$$

(30) remains valid for $d=1$ and $p=\kappa_{1}(d)=\infty$.

(ii) Let $d \geq 2$ and $t>0$. Then $\nabla Z(t)$ belongs to $L_{\frac{d}{d-1}, \infty}\left(\mathbb{R}^{d} ; \mathbb{R}^{d}\right)$, and we have

$$
|\nabla Z(t)|_{\frac{d}{d-1}, \infty} \lesssim t^{-\alpha}, \quad t>0
$$


Proof. The proof uses the gradient estimates for $Z$ from Proposition 3.1 and is analogous to that of Theorem 3.1 and Theorem 3.2, respectively.

By means of the $L_{p}\left(\mathbb{R}^{d}\right)$-estimates for $\nabla Z(t, x)$ from Theorem 3.4 we can derive temporal decay estimates for the gradient of the solution. We record these estimates in the following theorem. Using $\nabla u(t, \cdot)=\nabla Z(t, \cdot) \star u_{0}$ the proof is analogous to the one of Theorem 3.3.

Theorem 3.5 (i) Let $d \in \mathbb{N}, 1 \leq p<\kappa_{1}(d), 1 \leq q, r \leq \infty, 1+\frac{1}{r}=\frac{1}{p}+\frac{1}{q}$, and $u_{0} \in L_{q}\left(\mathbb{R}^{d}\right)$. Then for $u(t)=Z(t) \star u_{0}$ we have

$$
|\nabla u(t)|_{r} \lesssim t^{-\frac{\alpha}{2}-\frac{\alpha d}{2}\left(1-\frac{1}{p}\right)}, \quad t>0 .
$$

(ii) Let $d \geq 2,1<q, r<\infty, \frac{1}{r}+\frac{2}{d}=\frac{1}{q}$, and $u_{0} \in L_{q}\left(\mathbb{R}^{d}\right)$. Then for $u(t)=Z(t) \star u_{0}$ we have

$$
|\nabla u(t)|_{r} \lesssim t^{-\alpha}, \quad t>0 .
$$

(iii) Let $d \geq 2$ and $u_{0} \in L_{1}\left(\mathbb{R}^{d}\right)$. Then for $u(t)=Z(t) \star u_{0}$ we have

$$
|\nabla u(t)|_{\frac{d}{d-1}, \infty} \lesssim t^{-\alpha}, \quad t>0 .
$$

Arguing analogously to the proof of Corollary 3.1 we obtain

Corollary 3.3 Let $1<r<\infty, d \in \mathbb{N}, u_{0} \in L_{1}\left(\mathbb{R}^{d}\right) \cap L_{r}\left(\mathbb{R}^{d}\right)$, and $u(t)=Z(t) \star u_{0}$. Then

$$
\begin{aligned}
|\nabla u(t)|_{r} & \lesssim t^{-\frac{\alpha}{2}-\frac{\alpha d}{2}\left(1-\frac{1}{r}\right)}, \quad t>0, \quad \text { if } d=1, \\
|\nabla u(t)|_{r} & \lesssim t^{-\frac{\alpha}{2}-\frac{\alpha d}{2}\left(1-\frac{1}{r}\right)}, \quad t>0, \quad \text { if } d \geq 2 \text { and } d<\frac{r}{r-1}, \\
|\nabla u(t)|_{r, \infty} & \lesssim t^{-\alpha}, \quad t>0, \quad \text { if } d \geq 2 \text { and } d=\frac{r}{r-1}, \\
|\nabla u(t)|_{r} & \lesssim t^{-\alpha}, \quad t>0, \quad \text { if } d \geq 2 \text { and } d>\frac{r}{r-1} .
\end{aligned}
$$

In the important special case $r=2$ the picture is as follows.

Corollary 3.4 Let $d \in \mathbb{N}$ and $u_{0} \in L_{1}\left(\mathbb{R}^{d}\right) \cap L_{2}\left(\mathbb{R}^{d}\right)$ and $u(t)=Z(t) \star u_{0}$. Then

$$
\begin{aligned}
|\nabla u(t)|_{2} & \lesssim t^{-\min \left\{\frac{\alpha}{2}+\frac{\alpha d}{4}, \alpha\right\}}, \quad t>0, d \in \mathbb{N} \backslash\{2\}, \\
|\nabla u(t)|_{2, \infty} & \lesssim t^{-\alpha}, \quad t>0, d=2 .
\end{aligned}
$$

Corollary 3.3 shows a critical dimension phenomenon for the gradient estimates with critical dimension $d_{\text {grad,crit }}=r /(r-1)<d_{\text {crit }}$.

\subsection{Large time behaviour of $Z(t, \cdot) \star u_{0}$}

In this subsection, we want to show that for integrable initial data $u_{0}$ the asymptotic behaviour of $Z(t, \cdot) \star u_{0}$ as $t \rightarrow \infty$ is described by a multiple of $Z(t, x)$. The corresponding result for the heat equation is well-known, see e.g. [31, Prop. 48.6].

For our strategy of proof we need the following decomposition lemma from [11]. 
Lemma 3.1 Suppose $f \in L_{1}\left(\mathbb{R}^{d}\right)$ such that $\int_{\mathbb{R}^{d}}|x||f(x)| d x<\infty$. Then there exists $F \in$ $L_{1}\left(\mathbb{R}^{d} ; \mathbb{R}^{d}\right)$ such that

$$
f=\left(\int_{\mathbb{R}^{d}} f(x) d x\right) \delta_{0}+\operatorname{div} F
$$

in the distributional sense and

$$
|F|_{L_{1}\left(\mathbb{R}^{d} ; \mathbb{R}^{d}\right)} \leq C_{d} \int_{\mathbb{R}^{d}}|x||f(x)| d x
$$

We have now the following result.

Theorem 3.6 Let $d \in \mathbb{N}$ and $1 \leq p<\kappa_{1}(d)$. Let $u_{0} \in L_{1}\left(\mathbb{R}^{d}\right)$ and set $M=\int_{\mathbb{R}^{d}} u_{0}(y) d y$.

(i) There holds

$$
t^{\frac{\alpha d}{2}\left(1-\frac{1}{p}\right)}|u(t)-M Z(t)|_{p} \rightarrow 0, \quad \text { as } t \rightarrow \infty .
$$

(ii) Assume in addition that ||$x\left|u_{0}\right|_{1}<\infty$. Then

$$
t^{\frac{\alpha d}{2}\left(1-\frac{1}{p}\right)}|u(t)-M Z(t)|_{p} \lesssim t^{-\frac{\alpha}{2}}, \quad t>0 .
$$

Moreover, in the limit case $p=\kappa_{1}(d)$ we have

$$
t^{\frac{\alpha}{2}}|u(t)-M Z(t)|_{\kappa_{1}(d), \infty} \lesssim t^{-\frac{\alpha}{2}}, \quad t>0 .
$$

Proof. The strategy of the proof is the same as in [45, p. 14, 15].

(a) Suppose first that $u_{0} \in L_{1}\left(\mathbb{R}^{d}\right)$ is such that $\int_{\mathbb{R}^{d}}|x|\left|u_{0}(x)\right| d x<\infty$. By Lemma 3.1 there exists $\phi \in L_{1}\left(\mathbb{R}^{d} ; \mathbb{R}^{d}\right)$ such that

$$
u_{0}=M \delta_{0}+\operatorname{div} \phi
$$

and $|\phi|_{1} \leq C_{d}|| x\left|u_{0}\right|_{1}$. Consequently,

$$
\begin{aligned}
u(t, x) & =M\left(Z(t, \cdot) \star \delta_{0}\right)(x)+(Z(t, \cdot) \star \operatorname{div} \phi(\cdot))(x) \\
& =M Z(t, x)+(\nabla Z(t, \cdot) \star \phi)(x),
\end{aligned}
$$

which yields

$$
u(t, x)-M Z(t, x)=(\nabla Z(t, \cdot) \star \phi)(x) .
$$

By Young's inequality it follows that for any $1 \leq p<\kappa_{1}(d)$

$$
|u(t)-M Z(t)|_{p} \leq|\nabla Z(t)|_{p}|\phi|_{1} \lesssim|\nabla Z(t)|_{p}|| x\left|u_{0}\right|_{1} \lesssim t^{-\frac{\alpha}{2}-\frac{\alpha d}{2}\left(1-\frac{1}{p}\right)},
$$

where we used Theorem 3.4. Hence

$$
t^{\frac{\alpha d}{2}\left(1-\frac{1}{p}\right)}|u(t)-M Z(t)|_{p} \lesssim t^{-\frac{\alpha}{2}}
$$

which is the first part of assertion (ii). The second part follows from (31) by applying Young's inequality for weak $L_{p}$-spaces ([14, Theorem 1.2.13]). 
(b) To prove (i) we choose a sequence $\left(\eta_{j}\right) \subset C_{0}^{\infty}\left(\mathbb{R}^{d}\right)$ such that $\int_{\mathbb{R}^{d}} \eta_{j} d x=M$ for all $j$ and $\eta_{j} \rightarrow u_{0}$ in $L_{1}\left(\mathbb{R}^{d}\right)$. For each $j$ we have by Part (a) and by Theorem 3.1

$$
\begin{aligned}
|u(t)-M Z(t)|_{p} & \leq\left|Z(t) \star\left(u_{0}-\eta_{j}\right)\right|_{p}+\left|Z(t) \star \eta_{j}-M Z(t)\right|_{p} \\
& \leq|Z(t)|_{p}\left|u_{0}-\eta_{j}\right|_{1}+C(j) t^{-\frac{\alpha}{2}-\frac{\alpha d}{2}\left(1-\frac{1}{p}\right)} \\
& \leq C_{1} t^{-\frac{\alpha d}{2}\left(1-\frac{1}{p}\right)}\left|u_{0}-\eta_{j}\right|_{1}+C(j) t^{-\frac{\alpha}{2}-\frac{\alpha d}{2}\left(1-\frac{1}{p}\right)},
\end{aligned}
$$

and therefore

$$
t^{\frac{\alpha d}{2}\left(1-\frac{1}{p}\right)}|u(t)-M Z(t)|_{p} \leq C_{1}\left|u_{0}-\eta_{j}\right|_{1}+C(j) t^{-\frac{\alpha}{2}},
$$

which implies

$$
\limsup _{t \rightarrow \infty} t^{\frac{\alpha d}{2}\left(1-\frac{1}{p}\right)}|u(t)-M Z(t)|_{p} \leq C_{1}\left|u_{0}-\eta_{j}\right|_{1} .
$$

Assertion (i) follows by sending $j \rightarrow \infty$.

\section{General subdiffusion equations, $L_{2}$-estimates}

Let $(k, l) \in \mathcal{P C}$. Assuming that $u_{0} \in L_{1}\left(\mathbb{R}^{d}\right) \cap L_{2}\left(\mathbb{R}^{d}\right)$ we want to derive $L_{2}$-decay estimates for solutions of

$$
\partial_{t}\left(k *\left[u-u_{0}\right]\right)-\Delta u=0, \quad t>0, x \in \mathbb{R}^{d},
$$

together with

$$
\left.u\right|_{t=0}=u_{0}, x \in \mathbb{R}^{d},
$$

by means of harmonic analysis, the main tool being Plancherel's theorem.

Under appropriate assumptions on the initial value $u_{0}$ (see Kochubei [23]) the solution of (32), (33) is given by

$$
u(t, x)=\int_{\mathbb{R}^{N}} Z(t, x-y) u_{0}(y) d y .
$$

In the following we will assume that $u$ is defined by (34).

Theorem 4.1 Let $d \in \mathbb{N}$ and $u_{0} \in L_{1}\left(\mathbb{R}^{d}\right) \cap L_{2}\left(\mathbb{R}^{d}\right)$. Assume that $k$ satisfies condition $(\mathcal{P C})$ and that $u$ is given by (34). Assume further that $\widetilde{u_{0}}(0) \neq 0$. Then

$$
|u(t)|_{2} \gtrsim k(t)^{\min \left\{1, \frac{d}{4}\right\}}, \quad \text { a.a. } t \geq 1 .
$$

Proof. Let $\rho_{0}>0, t>0$, and $\rho=\rho(t) \in\left(0, \rho_{0}\right]$. By Plancherel's theorem and the monotonicity property of $s_{\mu}$ w.r.t. $\mu$ we have

$$
\begin{aligned}
(2 \pi)^{d}|u(t, \cdot)|_{2}^{2} & =|\tilde{u}(t, \cdot)|_{2}^{2}=\int_{\mathbb{R}^{d}} \tilde{Z}(t, \xi)^{2}\left|\widetilde{u_{0}}(\xi)\right|^{2} d \xi \geq \int_{B_{\rho}} \tilde{Z}(t, \xi)^{2}\left|\widetilde{u_{0}}(\xi)\right|^{2} d \xi \\
& =\int_{B_{\rho}} s\left(t,|\xi|^{2}\right)^{2}\left|\widetilde{u_{0}}(\xi)\right|^{2} d \xi \geq s\left(t, \rho^{2}\right)^{2} \int_{B_{\rho}}\left|\widetilde{u_{0}}(\xi)\right|^{2} d \xi \\
& =s\left(t, \rho^{2}\right)^{2} \rho^{d}\left(\rho^{-d} \int_{B_{\rho}}\left|\widetilde{u_{0}}(\xi)\right|^{2} d \xi\right) .
\end{aligned}
$$


Recall that $u_{0} \in L_{1}\left(\mathbb{R}^{d}\right) \cap L_{2}\left(\mathbb{R}^{d}\right)$ implies that $\widetilde{u_{0}} \in C_{0}\left(\mathbb{R}^{d}\right) \cap L_{2}\left(\mathbb{R}^{d}\right)$.

By the assumption $\widetilde{u_{0}}(0) \neq 0$, we may choose $\rho_{0}$ so small that we get an estimate

$$
\rho^{-d} \int_{B_{\rho}}\left|\widetilde{u_{0}}(\xi)\right|^{2} d \xi \geq c_{1} \quad \text { for all } \rho \in\left(0, \rho_{0}\right],
$$

with some constant $c_{1}>0$. Using (36) and the lower estimate in (11) we deduce from (35) that

$$
(2 \pi)^{d}|u(t, \cdot)|_{2}^{2} \geq \frac{c_{1} \rho^{d}}{\left(1+\rho^{2} k(t)^{-1}\right)^{2}} .
$$

We first choose

$$
\rho=\rho(t)=\frac{\rho_{0}}{\left(1+k(t)^{-1}\right)^{1 / 2}} .
$$

With this choice we have $\rho(t)^{2} k(t)^{-1} \leq \rho_{0}^{2}$ and thus

$$
\begin{aligned}
(2 \pi)^{d}|u(t, \cdot)|_{2}^{2} & \geq \frac{c_{1} \rho^{d}}{\left(1+\rho_{0}^{2}\right)^{2}}=\frac{c_{1} \rho_{0}^{d}}{\left(1+\rho_{0}^{2}\right)^{2}}\left(\frac{k(t)}{1+k(t)}\right)^{\frac{d}{2}} \\
& \geq \frac{c_{1} \rho_{0}^{d} k(t)^{d / 2}}{\left(1+\rho_{0}^{2}\right)^{2}\left(1+k\left(t_{0}\right)\right)^{d / 2}}, \quad t \geq t_{0}>0,
\end{aligned}
$$

since $k$ is nonincreasing. This shows that in this situation we have $|u(t)|_{2} \gtrsim k(t)^{d / 4}$ for $t \geq 1$.

Let us next choose $\rho=\rho(t)=\rho_{0}$. Then (37) yields

$$
(2 \pi)^{d}|u(t, \cdot)|_{2}^{2} \geq \frac{c_{1} \rho_{0}^{d}}{\left(1+\rho_{0}^{2} k(t)^{-1}\right)^{2}}=\frac{c_{1} \rho_{0}^{d} k(t)^{2}}{\left(k\left(t_{0}\right)+\rho_{0}^{2}\right)^{2}}, \quad t \geq t_{0}>0,
$$

and hence $|u(t)|_{2} \gtrsim k(t)$ for $t \geq 1$.

We turn now to upper estimates.

Theorem 4.2 Let $d \in \mathbb{N} \backslash\{4\}, u_{0} \in L_{1}\left(\mathbb{R}^{d}\right) \cap L_{2}\left(\mathbb{R}^{d}\right)$, and assume that $(k, l) \in \mathcal{P C}$. Suppose that $u$ is given by (34). Then there holds

$$
|u(t)|_{2} \lesssim[(1 * l)(t)]^{-\min \left\{1, \frac{d}{4}\right\}}, \quad t>0 .
$$

Proof. Suppose that $d \leq 3$. By Plancherel's theorem and the upper estimate in (11) we have

$$
\begin{aligned}
(2 \pi)^{d}|u(t, \cdot)|_{2}^{2} & =|\tilde{u}(t, \cdot)|_{2}^{2}=\int_{\mathbb{R}^{d}} s\left(t,|\xi|^{2}\right)^{2}\left|\widetilde{u_{0}}(\xi)\right|^{2} d \xi \leq\left|\widetilde{u_{0}}\right|_{\infty}^{2} \int_{\mathbb{R}^{d}} s\left(t,|\xi|^{2}\right)^{2} d \xi \\
& \leq C\left|u_{0}\right|_{1}^{2} \int_{\mathbb{R}^{d}} \frac{d \xi}{\left(1+|\xi|^{2}(1 * l)(t)\right)^{2}}=C_{1}\left|u_{0}\right|_{1}^{2} \int_{0}^{\infty} \frac{r^{d-1} d r}{\left(1+r^{2}(1 * l)(t)\right)^{2}} \\
& =\frac{C_{1}\left|u_{0}\right|_{1}^{2}}{((1 * l)(t))^{d / 2}} \int_{0}^{\infty} \frac{\rho^{d-1} d \rho}{\left(1+\rho^{2}\right)^{2}}=\frac{C_{2}\left|u_{0}\right|_{1}^{2}}{((1 * l)(t))^{d / 2}},
\end{aligned}
$$


which shows that $\left.|u(t)|_{2} \lesssim(1 * l)(t)\right)^{-d / 4}, t>0$. Observe that this estimate breaks down for $d \geq 4$, since the last integral becomes infinite.

Suppose now that $d \geq 5$. By interpolation it follows from $u_{0} \in L_{1}\left(\mathbb{R}^{d}\right) \cap L_{2}\left(\mathbb{R}^{d}\right)$ that $u_{0} \in L_{\frac{2 d}{d+4}}\left(\mathbb{R}^{d}\right)$. Since

$$
\frac{1}{\frac{2 d}{d+4}}-\frac{1}{2}=\frac{2}{d} \quad \text { and } \frac{2 d}{d+4}>1,
$$

the Hardy-Littlewood-Sobolev theorem on fractional integration, see e.g. [14, Thm. 6.1.3], implies $(-\Delta)^{-1} u_{0} \in L_{2}\left(\mathbb{R}^{d}\right)$. Using this property, Plancherel's theorem, and the upper estimate in (11) we may estimate as follows.

$$
\begin{aligned}
(2 \pi)^{d}|u(t, \cdot)|_{2}^{2} & =\left.\left.\int_{\mathbb{R}^{d}}|\xi|^{4} s\left(t,|\xi|^{2}\right)^{2}|| \xi\right|^{-2} \widetilde{u_{0}}(\xi)\right|^{2} d \xi \\
& \leq\left.\left.\frac{1}{((1 * l)(t))^{2}} \int_{\mathbb{R}^{d}} \frac{|\xi|^{4}((1 * l)(t))^{2}}{\left(1+|\xi|^{2}(1 * l)(t)\right)^{2}}|| \xi\right|^{-2} \widetilde{u_{0}}(\xi)\right|^{2} d \xi \\
& \leq\left.\left.\frac{1}{((1 * l)(t))^{2}} \int_{\mathbb{R}^{d}}|| \xi\right|^{-2} \widetilde{u_{0}}(\xi)\right|^{2} d \xi=\frac{(2 \pi)^{d}}{((1 * l)(t))^{2}}\left|(-\Delta)^{-1} u_{0}\right|_{2}^{2} .
\end{aligned}
$$

Hence $\left.|u(t)|_{2} \lesssim(1 * l)(t)\right)^{-1}, t>0$. The theorem is proved.

Remark 4.1 Comparing the results from Theorem 4.1 and Theorem 4.2 we see that the estimate from Theorem 4.2 is in general optimal provided that

$$
(1 * l)(t)^{-1} \leq C k(t), \quad t \geq T
$$

where $C, T$ are some positive constants. This property of the kernels $k, l$ is satisfied in many important examples, in particular in the time-fractional and ultraslow diffusion case, see Examples 4.1 and 4.3 below. However, there exist examples where (39) is violated, see e.g. Example 4.4, and thus Theorem 4.2 may not give the optimal decay rate. Recall that a key ingredient in the proof of Theorem 4.2 is the upper bound

$$
s_{\mu}(t) \leq \frac{1}{1+\mu(1 * l)(t)}, \quad t \geq 0, \mu \geq 0
$$

If (39) is violated, then (40) may not be sharp w.r.t. the decay rate. In fact, this is the case in Example 4.4 below. To improve the decay estimate from Theorem 4.2 one has to upgrade the estimate for $s_{\mu}$.

Suppose we have an estimate

$$
s_{\mu}(t) \leq \frac{C}{1+\mu \psi(t)}, \quad t \geq 0, \mu \geq 0,
$$

where $\psi: \mathbb{R}_{+} \rightarrow \mathbb{R}_{+}$is a continuous function (typically nondecreasing) and the constant $C$ is independent of $\mu$. Then it follows from the proof of Theorem 4.2 that

$$
|u(t)|_{2} \lesssim[\psi(t)]^{-\min \left\{1, \frac{d}{4}\right\}}, \quad t>0
$$


Example 4.1 The time-fractional case. We consider the pair

$$
(k, l)=\left(g_{1-\alpha}, g_{\alpha}\right), \quad \text { where } \alpha \in(0,1) .
$$

Recall that the Laplace transform of $g_{\beta}, \beta>0$, is given by $\widehat{g_{\beta}}(z)=z^{-\beta}, \operatorname{Re} z>0$, and so it is easy to see that $g_{\beta_{1}} * g_{\beta_{2}}=g_{\beta_{1}+\beta_{2}}$ for all $\beta_{1}, \beta_{2}>0$. In particular $(k, l) \in \mathcal{P C}$. We further have

$$
(1 * l)(t)=\left(1 * g_{\alpha}\right)(t)=g_{1+\alpha}(t)=\frac{t^{\alpha}}{\Gamma(1+\alpha)}
$$

and so both $k(t)$ and $(1 * l)(t)^{-1}$ are of the form $c t^{-\alpha}$ with some constant $c>0$. Invoking Theorem 4.2 reproduces the estimates from Corollary 3.2 for noncritical $d$, that is,

$$
|u(t)|_{2} \lesssim t^{-\alpha \min \left\{1, \frac{d}{4}\right\}}, \quad t>0, d \in \mathbb{N} \backslash\{4\} .
$$

Theorem 4.1 shows that this estimate is optimal in the general case.

Example 4.2 A sum of two fractional derivatives. Let $0<\alpha<\beta<1$ and

$$
k(t)=g_{1-\alpha}(t)+g_{1-\beta}(t), \quad t>0 .
$$

Clearly, $k$ is completely monotone and $k(0+)=\infty$, and so by Theorem 5.4 in Chapter 5 of [16], the kernel $k$ has a resolvent $l \in L_{1, l o c}\left(\mathbb{R}_{+}\right)$of the first kind, that is $k * l=1$ on $(0, \infty)$, and this resolvent is completely monotone as well. In particular $(k, l) \in \mathcal{P C}$. Observe that

$$
\widehat{1 * l}(z)=\frac{1}{z} \frac{1}{z^{\alpha}+z^{\beta}} \sim \frac{1}{z^{1+\alpha}} \quad \text { as } z \rightarrow 0,
$$

which yields $(1 * l)(t) \sim g_{1+\alpha}(t)$ as $t \rightarrow \infty$, by the Karamata-Feller Tauberian theorem, see [13]. It follows that there is $T_{1}>0$ such that $(1 * l)(t) \geq \frac{1}{2} g_{1+\alpha}(t)$ for all $t \geq T_{1}$. Applying Theorem 4.2 then yields

$$
|u(t)|_{2} \lesssim t^{-\alpha \min \left\{1, \frac{d}{4}\right\}}, \quad t \geq T_{1}, d \in \mathbb{N} \backslash\{4\} .
$$

Theorem 4.1 shows that this estimate is optimal. We see that the decay rate is determined by the fractional derivative of lower order. These considerations extend trivally to kernels $k(t)=$ $\sum_{j=1}^{m} \delta_{j} g_{1-\alpha_{j}}(t)$ with $\delta_{j}>0$ and $0<\alpha_{1}<\alpha_{2}<\ldots<\alpha_{m}<1$.

Example 4.3 The distributed order case (an example of ultraslow diffusion). We consider the pair

$$
k(t)=\int_{0}^{1} g_{\beta}(t) d \beta, \quad l(t)=\int_{0}^{\infty} \frac{e^{-s t}}{1+s} d s, \quad t>0 .
$$

Both kernels are nonnegative and nonincreasing, and there holds (see [41, Example 6.5])

$$
\hat{k}(z)=\frac{z-1}{z \log z}, \quad \hat{l}(z)=\frac{\log z}{z-1}, \quad \operatorname{Re} z>0 .
$$

Thus $(k, l) \in \mathcal{P C}$. There exists a number $T_{1}>1$ such that

$$
\frac{1}{2 k(t)} \leq \log t \leq 2(1 * l)(t), \quad t \geq T_{1},
$$


see [41, Example 6.5]. This together with Theorem 4.2 yields the logarithmic decay estimate

$$
|u(t)|_{2} \lesssim(\log t)^{-\min \left\{1, \frac{d}{4}\right\}}, \quad t \geq T_{1},
$$

which is optimal, by Theorem 4.1 .

Example 4.4 Switching the kernels from the previous example. We consider now the pair

$$
k(t)=\int_{0}^{\infty} \frac{e^{-s t}}{1+s} d s, \quad l(t)=\int_{0}^{1} g_{\beta}(t) d \beta, \quad, \quad t>0 .
$$

From the previous considerations we know already that $(k, l) \in \mathcal{P C}$. The kernel $k(t)$ in this example behaves like $t^{-1}$ as $t \rightarrow \infty$, whereas $(1 * l)(t) \sim t / \log t$ as $t \rightarrow \infty$, see [41, Example 6.6]. Thus $k(t)$ decays faster than $(1 * l)(t)^{-1}$, so that there is a gap between the decay rates provided by Theorem 4.1 and Theorem 4.2 . We claim that

$$
|u(t)|_{2} \lesssim t^{-\min \left\{1, \frac{d}{4}\right\}}, \quad t>0, d \in \mathbb{N} \backslash\{4\},
$$

which is optimal by Theorem 4.1. What is interesting here is that for $d \leq 3$ the decay rate is the same as for the heat equation!

The claim follows from the estimate

$$
s_{\mu}(t) \leq \frac{C}{1+\mu t}, \quad t \geq 0, \mu \geq 0,
$$

which has been shown in [41, Example 6.6], and from (42) in Remark 4.1. The proof of (43) in [41] is quite involved and makes use of Laplace transform methods.

\section{$5 \quad L_{r}$-estimates and the critical dimension case}

In this section we continue the study of the general subdiffusion problem (32), (33) under the assumption that the kernel $k$ is of type $\mathcal{P C}$.

Lemma 5.1 Assume that $(k, l) \in \mathcal{P C}$. Let $t \geq 0$ be fixed. Then the function $\mu \mapsto s(t, \mu)$ belongs to $C^{\infty}\left(\mathbb{R}_{+}\right)$and

$$
(-1)^{j} \partial_{\mu}^{j} s(t, \mu) \geq 0 \quad \text { for all } j \in \mathbb{N}_{0},
$$

in particular $s(t, \mu)$ is completely monotone w.r.t. $\mu$. Moreover,

$$
\mu^{j}\left|\partial_{\mu}^{j} s(t, \mu)\right| \leq 2^{j} j ! s\left(t, \frac{\mu}{2}\right) \quad \text { for all } j \in \mathbb{N}_{0} .
$$

Proof. Recall that $s(t, \mu)=s_{\mu}(t)$ solves the equation

$$
s_{\mu}(t)+\mu\left(s_{\mu} * l\right)(t)=1, \quad t, \mu \geq 0 .
$$

Since $\mu$ merely appears as coefficient in front of the second term, it is clear that the dependence of the solution $s_{\mu}(t)$ on the parameter $\mu$ is $C^{\infty}$. Differentiating w.r.t. $\mu$ gives

$$
\partial_{\mu} s_{\mu}+\mu\left(\partial_{\mu} s_{\mu} * l\right)+s_{\mu} * l=0,
$$


which is equivalent to the integro-differential equation

$$
\frac{d}{d t}\left(k * \partial_{\mu} s_{\mu}\right)(t)+\mu \partial_{\mu} s_{\mu}(t)=-s_{\mu}(t), \quad t>0, \quad \partial_{\mu} s_{\mu}(0)=0 .
$$

The property $\partial_{\mu} s_{\mu}(0)=0$ follows from the fact that $s_{\mu}(0)=1$ for all $\mu \geq 0$. Note also that $\left.\partial_{\mu} s_{\mu}\right|_{\mu=0}=-(1 * l)(t)$. Differentiating (46) w.r.t. $\mu$ leads to

$$
\frac{d}{d t}\left(k * \partial_{\mu}^{2} s_{\mu}\right)(t)+\mu \partial_{\mu}^{2} s_{\mu}(t)=-2 \partial_{\mu} s_{\mu}(t), \quad t>0, \quad \partial_{\mu}^{2} s_{\mu}(0)=0 .
$$

Differentiating further, a simple induction argument shows that

$$
\frac{d}{d t}\left(k * \partial_{\mu}^{j} s_{\mu}\right)(t)+\mu \partial_{\mu}^{j} s_{\mu}(t)=-j \partial_{\mu}^{j-1} s_{\mu}(t), \quad t>0, \quad \partial_{\mu}^{j} s_{\mu}(0)=0, \quad j \in \mathbb{N} .
$$

Assertion (44) follows then by means of induction from (47) and the fact that the solution $v$ of

$$
\frac{d}{d t}(k * v)(t)+\mu v(t)=f(t), \quad t>0, \quad v(0)=0,
$$

is nonnegative, whenever $f \in L_{1, l o c}\left(\mathbb{R}_{+}\right)$enjoys this property, see e.g. [41] for the latter property.

To see (45), we apply Taylor's theorem to the function $\mu \mapsto s(t, \mu)$, thereby obtaining that for every $n \in \mathbb{N}$

$$
s\left(t, \frac{\mu}{2}\right)=\sum_{j=0}^{n} \frac{\partial_{\mu}^{j} s(t, \mu)}{j !}\left(-\frac{\mu}{2}\right)^{j}+\frac{\partial_{\mu}^{n+1} s(t, \eta)}{(n+1) !}\left(-\frac{\mu}{2}\right)^{n+1},
$$

for some $\eta \in\left(\frac{\mu}{2}, \mu\right)$. In view of (44) every summand on the right-hand side of (48) is nonnegative. This implies

$$
s\left(t, \frac{\mu}{2}\right) \geq \frac{\partial_{\mu}^{j} s(t, \mu)}{j !}\left(-\frac{\mu}{2}\right)^{j} \quad \text { for all } j \leq n,
$$

which in turn yields (45).

Lemma 5.2 Let $(k, l) \in \mathcal{P C}$ and $t \geq 0$ be fixed. Let $\kappa \in(0,1]$ and set $\psi_{\kappa}(\mu)=\mu^{\kappa} s(t, \mu), \mu>0$. Then $\psi_{\kappa} \in C^{\infty}((0, \infty))$ and for every $n \in \mathbb{N}$ there exists a constant $C(n)>0$ such that

$$
\mu^{n}\left|\psi_{\kappa}^{(n)}(\mu)\right|[(1 * l)(t)]^{\kappa} \leq C(n), \quad \mu>0 .
$$

Proof. By Leibniz' formula for the $n$th derivative of a product of two functions we have

$$
\mu^{n} \psi_{\kappa}^{(n)}(\mu)=\sum_{j=0}^{n}\left(\begin{array}{l}
n \\
j
\end{array}\right)\left(\mu^{j} \partial_{\mu}^{j} s(t, \mu)\right) \cdot\left(\mu^{n-j} \partial_{\mu}^{n-j}\left(\mu^{\kappa}\right)\right),
$$

and thus by Lemma 5.1 and (11),

$$
\begin{aligned}
\mu^{n}\left|\psi_{\kappa}^{(n)}(\mu)\right| & \leq \sum_{j=0}^{n}\left(\begin{array}{c}
n \\
j
\end{array}\right)\left(\mu^{j}\left|\partial_{\mu}^{j} s(t, \mu)\right|\right) \cdot\left(\mu^{n-j}\left|\partial_{\mu}^{n-j}\left(\mu^{\kappa}\right)\right|\right) \\
& \leq \tilde{c}(n) \mu^{\kappa} \sum_{j=0}^{n}\left(\begin{array}{c}
n \\
j
\end{array}\right) 2^{j} j ! s\left(t, \frac{\mu}{2}\right) \\
& \leq C(n) \frac{\mu^{\kappa}}{1+\mu(1 * l)(t)} \leq \frac{C(n)}{[(1 * l)(t)]^{\kappa}} .
\end{aligned}
$$

This proves the lemma. 
Lemma 5.3 Let $(k, l) \in \mathcal{P C}, \delta \in(0,1]$, and $t \geq 0$ be fixed. Let $m_{0}(\xi)=\psi_{\delta}\left(|\xi|^{2}\right)=|\xi|^{2 \delta} s\left(t,|\xi|^{2}\right)$, $\xi \in \mathbb{R}^{d}$. Then $m_{0} \in C^{\infty}\left((0, \infty)^{d}\right)$ and for any multiindex $\beta=\left(\beta_{1}, \ldots, \beta_{d}\right) \in \mathbb{N}_{0}^{d}$ the partial derivative $\partial_{\xi}^{\beta} m_{0}$ of order $|\beta|=\sum_{i=1}^{d} \beta_{i}$ is a sum of finitely many terms of the form

$$
c(\beta) \cdot \psi_{\delta}^{(j)}\left(|\xi|^{2}\right) \cdot \prod_{i=1}^{d} \xi_{i}^{\gamma_{i}} \text { with } \frac{|\beta|}{2} \leq j \leq|\beta| \text { and } \sum_{i=1}^{d} \gamma_{i}=2 j-|\beta|
$$

where $c(\beta)>0$.

Moreover, the function $m(\xi):=m_{0}(\xi)[(1 * l)(t)]^{\delta}$ satisfies Mihlin's condition with a constant that is uniform w.r.t. $t \geq 0$, that is there exists $M=M(d)>0$ such that

$$
|\xi|^{|\beta|}\left|\partial_{\xi}^{\beta} m(\xi)\right| \leq M, \quad \xi \in \mathbb{R}^{d} \backslash\{0\},|\beta| \leq\left[\frac{d}{2}\right]+1 .
$$

Proof. The assertion on the structure of $\partial_{\xi}^{\beta} m_{0}$ can be proved by induction over $|\beta|$. If $|\beta|=0$, then $\partial_{\xi}^{\beta} m_{0}(\xi)=m_{0}(\xi)=\psi\left(|\xi|^{2}\right)$, which is of the desired form with $j=0$. Suppose now that the assertion is true for all $\beta \in \mathbb{N}_{0}^{d}$ of the same fixed order $b:=|\beta| \in \mathbb{N}_{0}$. Let $\beta^{\prime} \in \mathbb{N}_{0}^{d}$ with $\left|\beta^{\prime}\right|=b+1$. Then $\partial_{\xi}^{\beta^{\prime}} m_{0}=\partial_{\xi_{l}} \partial_{\xi}^{\beta} m_{0}$ for some $\beta \in \mathbb{N}_{0}^{d}$ with $|\beta|=b$ and some $l \in\{1, \ldots, d\}$. By the induction hypothesis, $\partial_{\xi_{l}} \partial_{\xi}^{\beta} m_{0}$ is a finite sum of first order partial derivatives w.r.t. $\xi_{l}$ of terms of the form described in (49). Let us consider such a term. If $\gamma_{l}=0$ we have

$$
\partial_{\xi_{l}}\left[\psi_{\delta}^{(j)}\left(|\xi|^{2}\right) \cdot \prod_{i=1}^{d} \xi_{i}^{\gamma_{i}}\right]=\psi_{\delta}^{(j+1)}\left(|\xi|^{2}\right) \cdot 2 \xi_{l} \cdot \prod_{i=1}^{d} \xi_{i}^{\gamma_{i}}
$$

whereas in case $\gamma_{l}>0$ we obtain

$$
\partial_{\xi_{l}}\left[\psi_{\delta}^{(j)}\left(|\xi|^{2}\right) \cdot \prod_{i=1}^{d} \xi_{i}^{\gamma_{i}}\right]=\psi_{\delta}^{(j+1)}\left(|\xi|^{2}\right) \cdot 2 \xi_{l} \cdot \prod_{i=1}^{d} \xi_{i}^{\gamma_{i}}+\psi_{\delta}^{(j)}\left(|\xi|^{2}\right) \cdot \gamma_{l} \xi_{l}^{\gamma_{l}-1} \prod_{i=1, i \neq l}^{d} \xi_{i}^{\gamma_{i}} .
$$

The first term on the right-hand side of (50) has the desired form, since with $\gamma_{i}^{\prime}:=\gamma_{i}, i \neq l$ and $\gamma_{l}^{\prime}=\gamma_{l}+1$, we have by the induction hypothesis

$$
0 \leq \sum_{i=1}^{d} \gamma_{i}^{\prime}=\sum_{i=1}^{d} \gamma_{i}+1=2 j-|\beta|+1=2(j+1)-\left|\beta^{\prime}\right| .
$$

The second term has the desired form as well, since setting $\gamma_{i}^{\prime}=\gamma_{i}, i \neq l$ and $\gamma_{l}^{\prime}=\gamma_{l}-1$, we have now

$$
0 \leq \sum_{i=1}^{d} \gamma_{i}^{\prime}=\sum_{i=1}^{d} \gamma_{i}-1=2 j-|\beta|-1=2 j-\left|\beta^{\prime}\right| .
$$


The second part of Lemma 5.3 follows from the first one and Lemma 5.2. In fact, for any term $T(\xi)$ of the form (49) Lemma 5.2 yields the estimate

$$
\begin{aligned}
|\xi|^{|\beta|}|T(\xi)| & \leq c(\beta)|\xi|^{|\beta|}\left|\psi_{\delta}^{(j)}\left(|\xi|^{2}\right)\right| \prod_{i=1}^{d}\left|\xi_{i}\right|^{\gamma_{i}} \\
& \leq c(\beta)|\xi|^{2 j}\left|\psi_{\delta}^{(j)}\left(|\xi|^{2}\right)\right| \cdot|\xi|^{|\beta|-2 j}|\xi|^{\sum_{i=1}^{d} \gamma_{i}} \\
& \leq \frac{C(\beta, j)}{[(1 * l)(t)]^{\delta}} .
\end{aligned}
$$

It is now evident that $m(\xi)$ satisfies Mihlin's condition with a constant $M$ that merely depends on the dimension $d$.

Relying on Lemma 5.3, we are now able to prove the following generalization of Theorem 3.3.

Theorem 5.1 Let $(k, l) \in \mathcal{P C}$ and suppose that $u$ is given by $u(t)=Z(t) \star u_{0}$, where $u_{0}$ is as described below.

(i) Let $d \in \mathbb{N}, 1<p<\kappa(d), 1<q, r<\infty, 1+\frac{1}{r}=\frac{1}{p}+\frac{1}{q}$, and $u_{0} \in L_{q}\left(\mathbb{R}^{d}\right)$. Then

$$
|u(t)|_{r} \lesssim[(1 * l)(t)]^{-\frac{d}{2}\left(1-\frac{1}{p}\right)}, \quad t>0 .
$$

(ii) Let $d \geq 3,1<q, r<\infty, \frac{1}{r}+\frac{2}{d}=\frac{1}{q}$, and $u_{0} \in L_{q}\left(\mathbb{R}^{d}\right)$. Then

$$
|u(t)|_{r} \lesssim[(1 * l)(t)]^{-1}, \quad t>0 .
$$

(iii) Let $d \geq 3$ and $u_{0} \in L_{1}\left(\mathbb{R}^{d}\right)$. Then

$$
|u(t)|_{\frac{d}{d-2}, \infty} \lesssim[(1 * l)(t)]^{-1}, \quad t>0 .
$$

Proof. (i) Set $\delta=\frac{d}{2}\left(1-\frac{1}{p}\right)$. If $d \leq 2$ it is clear that $\delta \in(0,1)$. If $d \geq 3$ we have by assumption $1<p<\kappa(d)=\frac{d}{d-2}$, which is equivalent to $\delta \in(0,1)$. With $t>0$ being fixed we write

$$
\tilde{u}(t, \xi)=[(1 * l)(t)]^{-\delta}\left(\psi_{\delta}\left(|\xi|^{2}\right)[(1 * l)(t)]^{\delta}\right)\left(|\xi|^{-2 \delta} \widetilde{u_{0}}(\xi)\right)
$$

By the Hardy-Littlewood-Sobolev theorem on fractional integration, see e.g. [14, Thm. 6.1.3], $(-\Delta)^{-\delta} u_{0} \in L_{r}\left(\mathbb{R}^{d}\right)$ and $\left|(-\Delta)^{-\delta} u_{0}\right|_{r} \leq C(d, \delta, q)\left|u_{0}\right|_{q}$; in fact, the choice of $\delta$ and the assumption $1+\frac{1}{r}=\frac{1}{p}+\frac{1}{q}$ imply that

$$
\frac{1}{q}-\frac{1}{r}=\frac{2 \delta}{d} \text { and } 2 \delta<d
$$

Thanks to Lemma 5.3 we know that $m(\xi)=\psi_{\delta}\left(|\xi|^{2}\right)[(1 * l)(t)]^{\delta}$ satisfies Mihlin's condition with a dimensional constant that is independent of $t>0$. Thus we may apply Mihlin's multiplier theorem, see [14, Theorem 5.2.7], thereby obtaining that

$$
|u(t)|_{r} \leq C(d, r)[(1 * l)(t)]^{-\delta}\left|(-\Delta)^{-\delta} u_{0}\right|_{r} \lesssim[(1 * l)(t)]^{-\delta} .
$$


This proves (i).

(ii) We consider again the decomposition (51), now setting $\delta=1$. As before we see that the Hardy-Littlewood-Sobolev theorem implies $(-\Delta)^{-1} u_{0} \in L_{r}\left(\mathbb{R}^{d}\right)$. The assertion follows then from Lemma 5.3 with $\delta=1$ and Mihlin's multiplier theorem.

(iii) We know already that $m(\xi)=\psi_{1}\left(|\xi|^{2}\right)[(1 * l)(t)]$ is an $L_{r}\left(\mathbb{R}^{d}\right)$-Fourier multiplier for all $r \in(1, \infty)$ with a constant that only depends on $r$ and $d$, that is, the operator $T$ defined by $T f=\mathcal{F}^{-1}(m \mathcal{F} f)\left(\mathcal{F}\right.$ denoting the Fourier transform) on a suitable dense subset of $L_{r}\left(\mathbb{R}^{d}\right)$ is $L_{r}\left(\mathbb{R}^{d}\right)$-bounded, thus extends to an operator $T \in \mathcal{B}\left(L_{r}\left(\mathbb{R}^{d}\right)\right)$, and $|T|_{\mathcal{B}\left(L_{r}\right)} \leq M(d, r)$. The weak $L_{r}$-spaces can be obtained from the strong ones by real interpolation. Assuming $1<r<\infty$ we may choose $r_{1} \in(1, r), r_{2} \in(r, \infty)$, and $\theta \in(0,1)$ such that $\frac{1}{r}=\frac{1-\theta}{r_{1}}+\frac{\theta}{r_{2}}$. By [37, Theorem 1.18.2], we then have $\left(L_{r_{1}}, L_{r_{2}}\right)_{\theta, \infty}=L_{r, \infty}$. It follows that $T \in \mathcal{B}\left(L_{r, \infty}\left(\mathbb{R}^{d}\right)\right)$, with a norm bound that only depends on $r$ and $d$.

We choose $r=\frac{d}{d-2}$. Then $1-\frac{1}{r}=\frac{2}{d}$, and the Hardy-Littlewood-Sobolev theorem ([14, Thm. 6.1.3]) implies that $(-\Delta)^{-1} u_{0} \in L_{r, \infty}\left(\mathbb{R}^{d}\right)$. Note that $u_{0} \in L_{1}\left(\mathbb{R}^{d}\right)$ and so we only get an estimate in a weak $L_{r}$-space. The assertion now follows from (51) with $\delta=1$ and the fact that $T \in \mathcal{B}\left(L_{r, \infty}\left(\mathbb{R}^{d}\right)\right)$, with a norm bound that is independent of $t>0$.

The following result generalizes Corollary 3.1 and is a direct consequence of the previous theorem. The proof is analogous to the one of Corollary 3.1.

Corollary 5.1 Let $(k, l) \in \mathcal{P C}, 1<r<\infty, d \in \mathbb{N}, u_{0} \in L_{1}\left(\mathbb{R}^{d}\right) \cap L_{r}\left(\mathbb{R}^{d}\right)$, and $u(t)=Z(t) \star u_{0}$. Then

$$
\begin{aligned}
|u(t)|_{r} & \lesssim[(1 * l)(t)]^{-\frac{d}{2}\left(1-\frac{1}{r}\right)}, \quad t>0, \quad \text { if } d<3, \\
|u(t)|_{r} & \lesssim[(1 * l)(t)]^{-\frac{d}{2}\left(1-\frac{1}{r}\right)}, \quad t>0, \quad \text { if } d \geq 3 \text { and } d<\frac{2 r}{r-1}, \\
|u(t)|_{r, \infty} & \lesssim[(1 * l)(t)]^{-1}, \quad t>0, \quad \text { if } d \geq 3 \text { and } d=\frac{2 r}{r-1}, \\
|u(t)|_{r} & \lesssim[(1 * l)(t)]^{-1}, \quad t>0, \quad \text { if } d \geq 3 \text { and } d>\frac{2 r}{r-1} .
\end{aligned}
$$

As a special case we obtain the expected weak $L_{2}$-decay estimate in the case of the critical dimension $d=4$, which was missing in Theorem 4.2 .

Corollary 5.2 Let $d=4, u_{0} \in L_{1}\left(\mathbb{R}^{d}\right)$, and assume that $(k, l) \in \mathcal{P C}$. Suppose that $u$ is given by $u(t)=Z(t) \star u_{0}$. Then

$$
|u(t)|_{2, \infty} \lesssim[(1 * l)(t)]^{-1}, \quad t>0 .
$$

Remark 5.1 Similarly as in Section 4 the decay results from this section might not be optimal if the condition (39) is violated, but can be improved provided one has an estimate (41) where $\psi(t)$ increases faster than $(1 * l)(t)$ as $t \rightarrow \infty$. In this case, the above statements from Lemma 5.2, Lemma 5.3, Theorem 5.1, Corollary 5.1, and Corollary 5.2 remain valid when $(1 * l)(t)$ is replaced by $\psi(t)$. 


\section{Decay estimates via the energy method}

In this section we study a time-fractional diffusion equation with a more general elliptic operator in divergence form. To be precise, we consider the problem

$$
\begin{aligned}
\partial_{t}^{\alpha}\left(u-u_{0}\right)-\operatorname{div}(A(t, x) \nabla u) & =0, \quad t>0, x \in \mathbb{R}^{d}, \\
\left.u\right|_{t=0} & =u_{0}, \quad x \in \mathbb{R}^{d} .
\end{aligned}
$$

Here, we assume $\alpha \in(0,1), u_{0} \in L_{1}\left(\mathbb{R}^{d}\right) \cap L_{2}\left(\mathbb{R}^{d}\right)$, and

(H) $A \in L_{\infty}\left((0, T) \times B_{R} ; \mathbb{R}^{d \times d}\right)$ for all $T, R>0$, and $\exists \nu>0$ such that

$$
(A(t, x) \xi \mid \xi) \geq \nu|\xi|^{2}, \quad \text { for a.a. }(t, x) \in(0, \infty) \times \mathbb{R}^{d}, \text { and all } \xi \in \mathbb{R}^{d},
$$

where $B_{R}=B_{R}(0)$ denotes the ball of radius $R$ centered at 0 . Let $\stackrel{\circ}{H}_{2}^{1}\left(B_{R}\right)=\overline{C_{0}^{\infty}\left(B_{R}\right)} H_{2}^{1}\left(B_{R}\right)$. We say that a function $u:(0, \infty) \times \mathbb{R}^{d} \rightarrow \mathbb{R}$ is a global weak solution of (52), (53) if for any $T, R>0$,

$$
\begin{aligned}
& \left.u\right|_{(0, T) \times B_{R}} \in\left\{v \in L_{2}\left([0, T] ; H_{2}^{1}\left(B_{R}\right)\right)\right. \text { such that } \\
& \left.\qquad g_{1-\alpha} * v \in C\left([0, T] ; L_{2}\left(B_{R}\right)\right), \text { and }\left.\left(g_{1-\alpha} * v\right)\right|_{t=0}=0\right\},
\end{aligned}
$$

and for any test function

$$
\eta \in H_{2}^{1}\left([0, T] ; L_{2}\left(B_{R}\right)\right) \cap L_{2}\left([0, T] ; \stackrel{\circ}{H}_{2}^{1}\left(B_{R}\right)\right)
$$

with $\left.\eta\right|_{t=T}=0$ there holds

$$
\int_{0}^{T} \int_{B_{R}}\left(-\eta_{t}\left[g_{1-\alpha} *\left(u-u_{0}\right)\right]+(A \nabla u \mid \nabla \eta)\right) d x d t=0
$$

Theorem 6.1 Let $d \in \mathbb{N}, \alpha \in(0,1), u_{0} \in L_{1}\left(\mathbb{R}^{d}\right) \cap L_{2}\left(\mathbb{R}^{d}\right)$, and suppose that condition $(\mathcal{H})$ is satisfied. Let $u$ be a global weak solution of (52), (53), and assume in addition that

$$
|\nabla u|, u^{2} \in L_{1, l o c}\left([0, \infty) ; L_{1}\left(\mathbb{R}^{d}\right)\right) .
$$

Then

$$
|u(t)|_{2} \lesssim t^{-\frac{\alpha d}{d+4}}, \quad t>0 .
$$

An important tool in our proof is the so-called fundamental identity for integro-differential operators of the form $\frac{d}{d t}(k * \cdot)$, cf. also [42]. It can be viewed as the analogue to the chain rule $(H(u))^{\prime}=H^{\prime}(u) u^{\prime}$.

Lemma 6.1 Let $T>0$ and $U$ be an open subset of $\mathbb{R}$. Let further $k \in H_{1}^{1}([0, T]), H \in C^{1}(U)$, and $u \in L_{1}([0, T])$ with $u(t) \in U$ for a.a. $t \in(0, T)$. Suppose that the functions $H(u), H^{\prime}(u) u$, and $H^{\prime}(u)(\dot{k} * u)$ belong to $L_{1}([0, T])$ (which is the case if, e.g., $u \in L_{\infty}([0, T])$ ). Then we have for a.a. $t \in(0, T)$,

$$
\begin{aligned}
H^{\prime}(u(t)) & \frac{d}{d t}(k * u)(t)=\frac{d}{d t}(k * H(u))(t)+\left(-H(u(t))+H^{\prime}(u(t)) u(t)\right) k(t) \\
& +\int_{0}^{t}\left(H(u(t-s))-H(u(t))-H^{\prime}(u(t))[u(t-s)-u(t)]\right)[-\dot{k}(s)] d s
\end{aligned}
$$


The lemma follows from a straightforward computation. We remark that (56) remains valid for singular kernels $k$, like e.g. $k=g_{1-\alpha}$ with $\alpha \in(0,1)$, provided that $u$ is sufficiently smooth.

The following result is new and a very useful implication of the fundamental identity.

Corollary 6.1 Let $T, U, k, H$, and $u$ be as in Lemma 6.1. Let $u_{0} \in U$, and assume in addition that $k$ is nonnegative and nonincreasing and that $H$ is convex. Then

$$
H^{\prime}(u(t)) \frac{d}{d t}\left(k *\left[u-u_{0}\right]\right)(t) \geq \frac{d}{d t}\left(k *\left[H(u)-H\left(u_{0}\right)\right]\right)(t), \quad \text { a.a. } t \in(0, T) .
$$

Proof. By the fundamental identity, convexity of $H$, and the properties of $k$, we have

$$
\begin{aligned}
H^{\prime}(u(t)) \frac{d}{d t} & \left(k *\left[u-u_{0}\right]\right)(t)=H^{\prime}(u(t)) \frac{d}{d t}(k * u)(t)-H^{\prime}(u(t)) u_{0} k(t) \\
& \geq \frac{d}{d t}(k * H(u))(t)+\left(-H(u(t))+H^{\prime}(u(t))\left[u(t)-u_{0}\right]\right) k(t) \\
& \geq \frac{d}{d t}(k * H(u))(t)-H\left(u_{0}\right) k(t) \\
& =\frac{d}{d t}\left(k *\left[H(u)-H\left(u_{0}\right)\right]\right)(t), \quad \text { a.a. } t \in(0, T),
\end{aligned}
$$

which shows the desired inequality.

An important consequence of Lemma 6.1 is the so-called $L_{p}$-norm inequality for operators of the form $\partial_{t}(k * \cdot)$, which has been established recently in [41]. Specializing to our situation $(p=2)$ it says the following.

Lemma 6.2 Let $T>0$ and $\Omega \subset \mathbb{R}^{d}$ be an open set. Let $k \in H_{1, \text { loc }}^{1}\left(\mathbb{R}_{+}\right)$be nonnegative and nonincreasing. Then for any $v \in L_{2}((0, T) \times \Omega)$ and any $v_{0} \in L_{2}(\Omega)$ there holds

$$
\int_{\Omega} v \partial_{t}\left(k *\left[v-v_{0}\right]\right) d x \geq|v(t)|_{L_{2}(\Omega)} \partial_{t}\left(k *\left[|v|_{L_{2}(\Omega)}-\left|v_{0}\right|_{L_{2}(\Omega)}\right]\right)(t), \quad \text { a.a.t } \in(0, T) .
$$

Proof. For the reader's convenience we give a proof for (58), which is also simpler than that in the more general case considered in [41].

By the fundamental identity, applied twice, Fubini's theorem, and the triangle inequality for the $L_{2}(\Omega)$-norm we have for a.a. $t \in(0, T)$

$$
\begin{aligned}
\int_{\Omega} v \partial_{t}(k * v) d x= & \int_{\Omega}\left(\frac{1}{2} \partial_{t}\left(k * v^{2}\right)+\frac{1}{2} k(t) v^{2}\right) d x \\
& +\int_{0}^{t} \int_{\Omega}|v(t, x)-v(t-s, x)|^{2} d x[-\dot{k}(s)] d s \\
\geq & \frac{1}{2} \partial_{t}\left(k *|v|_{L_{2}(\Omega)}^{2}\right)+\frac{1}{2} k(t)|v(t)|_{L_{2}(\Omega)}^{2} \\
& +\frac{1}{2} \int_{0}^{t}\left(|v(t)|_{L_{2}(\Omega)}-|v(t-s)|_{L_{2}(\Omega)}\right)^{2}[-\dot{k}(s)] d s \\
= & |v(t)|_{L_{2}(\Omega)} \partial_{t}\left(k *|v|_{L_{2}(\Omega)}\right)(t)
\end{aligned}
$$


From this and Hölder's inequality, we infer that for a.a. $t \in(0, T)$

$$
\begin{aligned}
\int_{\Omega} v \partial_{t}\left(k *\left[v-v_{0}\right]\right) d x & =\int_{\Omega} v \partial_{t}(k * v) d x-k(t) \int_{\Omega} v v_{0} d x \\
& \geq|v(t)|_{2} \partial_{t}\left(k *|v|_{2}\right)(t)-k(t)|v(t)|_{2}\left|v_{0}\right|_{2} \\
& =|v(t)|_{2} \partial_{t}\left(k *\left[|v|_{2}-\left|v_{0}\right|_{2}\right]\right)(t) .
\end{aligned}
$$

This proves the lemma.

We are now in position to prove Theorem 6.1.

Proof. 1. Regularized weak formulation. For $\mu>0$, define the kernel $h_{\mu} \in L_{1, l o c}\left(\mathbb{R}_{+}\right)$via the Volterra integral equation

$$
h_{\mu}(t)+\mu\left(h_{\mu} * g_{\alpha}\right)(t)=\mu g_{\alpha}(t), \quad t>0,
$$

and set $g_{1-\alpha, \mu}=g_{1-\alpha} * h_{\mu}$. It is well known that $g_{1-\alpha, \mu}$ is positive and nonincreasing, it belongs to $H_{1, l o c}^{1}([0, \infty))$, and the Yosida approximation $B_{n}=n B(n+B)^{-1}, n \in \mathbb{N}$, of the operator $B:=\partial_{t}^{\alpha}$ defined in an appropriate space takes the form $B_{n}=\partial_{t}\left(g_{1-\alpha, n} * \cdot\right)$, and $B_{n} \rightarrow B$ as $n \rightarrow \infty$. Further, $h_{\mu}$ is nonnegative for all $\mu>0$, and for $1 \leq p<\infty$ and $f \in L_{p}([0, T])$, we have $h_{n} * f \rightarrow f$ in $L_{p}([0, T])$ as $n \rightarrow \infty$. We refer to [40] and [43] for more background on $B_{n}$. Using these properties of the kernels $g_{1-\alpha, \mu}$ one can derive an equivalent weak formulation where the singular kernel $g_{1-\alpha}$ is replaced by the more regular kernels $g_{1-\alpha, n}$. In fact, it follows from the above definition of weak solution that for any $R>0$ and any function $\psi \in \stackrel{\circ}{H}_{2}^{1}\left(B_{R}\right)$ there holds

$$
\int_{B_{R}}\left(\psi \partial_{t}\left[g_{1-\alpha, n} *\left(u-u_{0}\right)\right]+\left(h_{n} *[A \nabla u] \mid \nabla \psi\right)\right) d x=0, \quad \text { a.a. } t>0, n \in \mathbb{N},
$$

cf. [43].

2. Positive and negative part. Denote by $y_{+}$and $y_{-}:=[-y]_{+}$the positive and negative part, respectively, of $y \in \mathbb{R}$. Appealing to [41, Lemma 4.1], it follows from Step 1 that for any $R>0$ and any nonnegative function $\psi \in \dot{H}_{2}^{1}\left(B_{R}\right)$,

$$
\int_{B_{R}}\left(\psi \partial_{t}\left[g_{1-\alpha, n} *\left(u_{+(-)}-\left[u_{0}\right]_{+(-)}\right)\right]+\left(h_{n} *\left[A \nabla\left(u_{+(-)}\right)\right] \mid \nabla \psi\right)\right) d x=0, \quad \text { a.a. } t>0, n \in \mathbb{N} \text {. }
$$

3. An $L_{1}\left(\mathbb{R}^{d}\right)$-bound for $u(t, \cdot)$. Letting $R>1$ we choose a nonnegative $\psi \in C_{0}^{1}\left(B_{R}\right)$ such that $\psi \equiv 1$ in $B_{R-1}$ and $\psi \leq 1$ as well as $|\nabla \psi| \leq 2$ in $B_{R}$. Assuming $t \in(0, T)$ and setting $\Lambda=|A|_{L_{\infty}\left((0, T) \times B_{R}\right)}$, where $T>0$ is arbitrarily fixed, (60) implies that for a.a. $t \in(0, T)$

$$
\partial_{t}\left(g_{1-\alpha, n} * \int_{B_{R}} \psi u_{+} d x\right) \leq \int_{B_{R}} g_{1-\alpha, n}(t)\left(u_{0}\right)_{+} \psi d x+2 \Lambda \int_{B_{R} \backslash B_{R-1}}\left|\nabla\left(u_{+}\right)\right| d x+\rho_{n}(t),
$$

where

$$
\rho_{n}(t)=\int_{B_{R}}\left(A \nabla\left(u_{+}\right)-h_{n} *\left[A \nabla\left(u_{+}\right)\right] \mid \nabla \psi\right) d x .
$$


Note that the term in brackets on the left-hand side of (61) vanishes at $t=0$. Thus convolving (61) with $g_{\alpha}$ and employing that $g_{\alpha} * g_{1-\alpha, n}=1 * h_{n}$, we obtain

$$
h_{n} * \int_{B_{R}} \psi u_{+} d x \leq\left(1 * h_{n}\right)(t) \int_{B_{R}} \psi\left(u_{0}\right)_{+} d x+2 \Lambda g_{\alpha} * \int_{B_{R} \backslash B_{R-1}}\left|\nabla\left(u_{+}\right)\right| d x+\left(g_{\alpha} * \rho_{n}\right)(t),
$$

Next, sending $n \rightarrow \infty$, using the approximation property of the kernels $h_{n}$, and restricting to a subsequence, if necessary, we find that

$$
\int_{B_{R-1}} u_{+} d x \leq \int_{B_{R}} \psi\left(u_{0}\right)_{+} d x+2 \Lambda g_{\alpha} * \int_{\mathbb{R}^{d} \backslash B_{R-1}}\left|\nabla\left(u_{+}\right)\right| d x,
$$

for a.a. $t \in(0, T)$. By (54) and Young's inequality, the second term on the right-hand side of (62) tends to 0 in $L_{1}([0, T])$ as $R \rightarrow \infty$. Thus, sending $R \rightarrow \infty$ in (62) implies

$$
\left|u_{+}(t)\right|_{L_{1}\left(\mathbb{R}^{d}\right)} \leq\left|\left(u_{0}\right)_{+}\right|_{L_{1}\left(\mathbb{R}^{d}\right)}, \quad \text { a.a. } t \in(0, T) .
$$

The same argument leads to the corresponding estimate for $u_{-}$. Since $T>0$ was arbitrary, we conclude that $|u(t)|_{1} \leq 2\left|u_{0}\right|_{1}$ for a.a. $t>0$.

4. A fractional differential inequality for the $L_{2}\left(\mathbb{R}^{d}\right)$-norm. Let $R>1$ and $\psi$ be as in Step 3. For $t>0$ we take in (59) with $\psi$ replaced by $\eta$ the test function $\eta=u \psi^{2}$. This gives

$$
\int_{B_{R}}\left(\psi u \partial_{t}\left[g_{1-\alpha, n} *\left(\psi u-\psi u_{0}\right)\right]+\left(A \nabla u \mid \nabla\left(u \psi^{2}\right)\right)\right) d x=\rho_{n, R}(t), \quad \text { a.a. } t>0, n \in \mathbb{N},
$$

where

$$
\rho_{n, R}(t)=\int_{B_{R}}\left(A \nabla u-h_{n} *[A \nabla u] \mid \nabla \eta\right) d x .
$$

Assume $t \in(0, T)$ and set $\Lambda=|A|_{L_{\infty}\left((0, T) \times B_{R}\right)}$, where $T>0$ is arbitrarily fixed. By assumption $(\mathcal{H})$, we may estimate pointwise a.e. as follows

$$
\begin{aligned}
\left(A \nabla u \mid \nabla\left(u \psi^{2}\right)\right) & =(A \nabla u \mid \nabla u) \psi^{2}+2(A \nabla u \mid u \psi \nabla \psi) \\
& \geq \nu|\nabla u|^{2} \psi^{2}-\frac{\nu}{2}|\nabla u|^{2} \psi^{2}-\frac{2 \Lambda^{2}}{\nu} u^{2}|\nabla \psi|^{2} \\
& \geq \frac{\nu}{2}|\nabla(u \psi)-u \nabla \psi|^{2}-\frac{2 \Lambda^{2}}{\nu} u^{2}|\nabla \psi|^{2} \\
& \geq \frac{\nu}{4}|\nabla(u \psi)|^{2}-\left(\frac{\nu}{2}+\frac{2 \Lambda^{2}}{\nu}\right) u^{2}|\nabla \psi|^{2} .
\end{aligned}
$$

The first term in (63) can be estimated from below by means of Lemma 6.2. Together with the preceding inequality we then obtain

$$
\begin{gathered}
|(\psi u)(t)|_{L_{2}\left(B_{R}\right)} \partial_{t}\left(g_{1-\alpha, n} *\left[|\psi u|_{L_{2}\left(B_{R}\right)}-\left|\psi u_{0}\right|_{L_{2}\left(B_{R}\right)}\right]\right)(t)+\frac{\nu}{4} \int_{B_{R}}|\nabla(u \psi)|^{2} d x \\
\leq C(\nu, \Lambda) \int_{B_{R}} u^{2}|\nabla \psi|^{2} d x+\rho_{n, R}(t) \\
\leq 4 C(\nu, \Lambda) \int_{B_{R} \backslash B_{R-1}} u^{2} d x+\rho_{n, R}(t), \quad \text { a.a. } t \in(0, T) .
\end{gathered}
$$


By Nash's inequality (cf. [29], [32]) and Step 3, we have for $v=\psi u$ (with $R$ being fixed)

$$
\begin{aligned}
|v(t)|_{L_{2}\left(\mathbb{R}^{d}\right)}^{2+\frac{4}{d}} & \leq C(d)|v(t)|_{L_{1}\left(\mathbb{R}^{d}\right)}^{\frac{4}{d}}|\nabla v(t)|_{L_{2}\left(\mathbb{R}^{d}\right)}^{2} \\
& \leq C(d)\left(2\left|u_{0}\right|_{L_{1}\left(\mathbb{R}^{d}\right)}\right)^{\frac{4}{d}}|\nabla v(t)|_{L_{2}\left(\mathbb{R}^{d}\right)}^{2} .
\end{aligned}
$$

Since $\psi$ vanishes outside $B_{R}$, we thus obtain

$$
\begin{array}{r}
|v(t)|_{L_{2}\left(\mathbb{R}^{d}\right)} \partial_{t}\left(g_{1-\alpha, n} *\left[|v|_{L_{2}\left(\mathbb{R}^{d}\right)}-\left|\psi u_{0}\right|_{L_{2}\left(\mathbb{R}^{d}\right)}\right]\right)(t)+\mu|v(t)|_{L_{2}\left(\mathbb{R}^{d}\right)}^{2+\frac{4}{d}} \\
\leq 4 C(\nu, \Lambda) \int_{\mathbb{R}^{d} \backslash B_{R-1}} u^{2} d x+\rho_{n, R}(t), \quad \text { a.a. } t \in(0, T),
\end{array}
$$

for some constant $\mu=\mu\left(\nu, d,\left|u_{0}\right|_{L_{1}\left(\mathbb{R}^{d}\right)}\right)>0$. Letting $\varepsilon>0$, we next divide by $|v(t)|_{L_{2}\left(\mathbb{R}^{d}\right)}+\varepsilon$. By Corollary 6.1, applied to $k=g_{1-\alpha, n}$ and the convex function $H(y)=y-\varepsilon \log (y+\varepsilon), y>-\varepsilon$, with derivative $H^{\prime}(y)=1-\frac{\varepsilon}{y+\varepsilon}=\frac{y}{y+\varepsilon}$ it follows that

$$
\begin{aligned}
& \partial_{t}\left(g_{1-\alpha, n} *\left[|v|_{L_{2}\left(\mathbb{R}^{d}\right)}-\left|\psi u_{0}\right|_{L_{2}\left(\mathbb{R}^{d}\right)}\right]\right)(t)+\mu \frac{|v(t)|_{L_{2}\left(\mathbb{R}^{d}\right)}^{2+\frac{4}{d}}}{|v(t)|_{L_{2}\left(\mathbb{R}^{d}\right)}+\varepsilon} \\
& \leq \frac{4 C(\nu, \Lambda)}{|v(t)|_{L_{2}\left(\mathbb{R}^{d}\right)}+\varepsilon} \int_{\mathbb{R}^{d} \backslash B_{R-1}} u^{2} d x+\frac{\rho_{n, R}(t)}{|v(t)|_{L_{2}\left(\mathbb{R}^{d}\right)}+\varepsilon} \\
& \quad+\varepsilon \partial_{t}\left(g_{1-\alpha, n} *\left(\log \left(|v|_{L_{2}\left(\mathbb{R}^{d}\right)}+\varepsilon\right)-\log \left(\left|\psi u_{0}\right|_{L_{2}\left(\mathbb{R}^{d}\right)}+\varepsilon\right)\right), \quad \text { a.a.t } \in(0, T) .\right.
\end{aligned}
$$

Next, let $\varphi \in C^{1}([0, T])$ be nonnegative and such that $\varphi(T)=0$. We multiply the preceding inequality by $\varphi(t)$, integrate on $(0, T)$, and integrate by parts (first and last term). Sending then $n \rightarrow \infty$, the term involving $\rho_{n, R}$ drops. Sending next $R \rightarrow \infty$ and recalling the dependence of $\psi$ (and thus also of $v$ ) on $R$, the term involving $\int_{\mathbb{R}^{d} \backslash B_{R-1}} u^{2} d x$ goes to zero (by assumption (54)) and we obtain

$$
\begin{aligned}
& \int_{0}^{T}\left(-\varphi_{t} g_{1-\alpha} *\left[|u|_{L_{2}\left(\mathbb{R}^{d}\right)}-\left|u_{0}\right|_{L_{2}\left(\mathbb{R}^{d}\right)}\right]+\mu \varphi \frac{|u|_{L_{2}\left(\mathbb{R}^{d}\right)}^{2+\frac{4}{d}}}{|u|_{L_{2}\left(\mathbb{R}^{d}\right)}+\varepsilon}\right) d t \\
& \quad \leq \varepsilon \int_{0}^{T}\left(-\varphi_{t} g_{1-\alpha} *\left(\log \left(|u|_{L_{2}\left(\mathbb{R}^{d}\right)}+\varepsilon\right)-\log \left(\left|u_{0}\right|_{L_{2}\left(\mathbb{R}^{d}\right)}+\varepsilon\right)\right)\right) d t \\
& \quad \leq 2 \varepsilon|\log \varepsilon| \int_{0}^{T}\left|\varphi_{t}\right| g_{2-\alpha} d t
\end{aligned}
$$

Sending now $\varepsilon \rightarrow 0$ the right-hand side drops, and we conclude that the $L_{2}\left(\mathbb{R}^{d}\right)$-norm of $u(t, \cdot)$ satisfies the fractional differential inequality

$$
\partial_{t}^{\alpha}\left(|u|_{2}-\left|u_{0}\right|_{2}\right)(t)+\mu|u(t)|_{2}^{1+\frac{4}{d}} \leq 0, \quad t \in(0, T),
$$

in the weak sense.

5. Comparison principle and decay estimate. By the comparison principle for timefractional differential equations (see [41, Lemma 2.6 and Remark 2.1]), (64) implies that $|u(t)|_{2} \leq$ $w(t)$ for a.a. $t \in(0, T)$, where $w$ solves the equation corresponding to $(64)$, that is

$$
\partial_{t}^{\alpha}\left(w-w_{0}\right)(t)+\mu w(t)^{\gamma}=0, \quad t>0, \quad w(0)=w_{0}:=\left|u_{0}\right|_{2},
$$


where we put $\gamma=1+\frac{4}{d}$. It is known that for $w_{0}>0$ there exist constants $c_{1}, c_{2}>0$ such that

$$
\frac{c_{1}}{1+t^{\frac{\alpha}{\gamma}}} \leq w(t) \leq \frac{c_{2}}{1+t^{\frac{\alpha}{\gamma}}}, \quad t \geq 0
$$

see [41, Theorem 7.1]. Since $T>0$ was arbitrary, we conclude that

$$
|u(t)|_{2} \leq w(t) \leq \frac{c_{2}}{1+t^{\frac{\alpha}{\gamma}}}=\frac{c_{2}}{1+t^{\frac{\alpha d}{d+4}}}, \quad \text { a.a. } t>0 .
$$

This finishes the proof of Theorem 6.1.

Note that the decay rate for the $L_{2}$-norm in (55) is strictly less than the one we obtained in Section 3, see (29). However, sending $d \rightarrow \infty$ the decay rate in (55) becomes $\alpha$, which is precisely the decay rate for the $L_{2}$-norm in (29) for $d>4$. This phenomenon of a smaller decay rate in the variational setting does not occur in the case $\alpha=1$. In fact, proceeding similarly as above in the latter case one obtains the differential inequality

$$
\partial_{t}|u(t)|_{2}+\mu|u(t)|_{2}^{\gamma} \leq 0, \quad t>0
$$

with the same $\gamma(>1)$ and $\mu$ as before. This implies

$$
|u(t)|_{2} \lesssim t^{-\frac{1}{\gamma-1}}=t^{-\frac{d}{4}} .
$$

On the other hand, the $L_{p}\left(\mathbb{R}^{d}\right)$-norm of the Gaussian heat kernel $H(t, x)=(4 \pi t)^{-d / 2} \exp \left(-\frac{|x|^{2}}{4 t}\right)$ decays as

$$
|H(t)|_{p} \lesssim t^{-\frac{d}{2}\left(1-\frac{1}{p}\right)}, \quad t>0,1 \leq p \leq \infty .
$$

Assuming $u_{0} \in L_{1}\left(\mathbb{R}^{d}\right)$, Young's inequality implies that the $L_{2}\left(\mathbb{R}^{d}\right)$-norm of $u(t)=H(t) \star u_{0}$ decays as

$$
|u(t)|_{2} \lesssim t^{-\frac{d}{2}\left(1-\frac{1}{2}\right)}=t^{-\frac{d}{4}}, \quad t>0,
$$

which is precisely the decay rate we obtain by means of energy estimates. It is an interesting open problem whether the decay rate in (55) is optimal in general. If this was not the case how could it be upgraded?

\section{References}

[1] Allen, M.; Caffarelli, L.; Vasseur, A.: A parabolic problem with a fractional time derivative. Available online at http://arxiv.org/pdf/1501.07211.pdf

[2] Bjorland, C.; Schonbek, M. E.: Poincaré's inequality and diffusive evolution equations. Adv. Differential Equations 14 (2009), 241-260.

[3] Brändle, C.; de Pablo, A.: Decay estimates for linear and nonlinear nonlocal heat equations. Available online at http://arxiv.org/pdf/1312.4661v1.pdf

[4] Bouchaud, J.-Ph.; Georges, A.: Anomalous diffusion in disordered media: statistical mechanisms, models and physical applications. Phys. Rep. 195 (1990), 127-293.

[5] Caffarelli, L.; Vazquez, J. L.: Asymptotic behaviour of a porous medium equation with fractional diffusion. Discrete Contin. Dyn. Syst. 29 (2011), 1393-1404. 
[6] Caputo, M.: Diffusion of fluids in porous media with memory. Geothermics 28 (1999), 113-130.

[7] Chasseigne, E.; Chaves, M.; Rossi, J. D.: Asymptotic behavior for nonlocal diffusion equations. J. Math. Pures Appl. 86 (2006), 271-291.

[8] Clément, Ph.; Nohel, J.A.: Abstract linear and nonlinear Volterra equations preserving positivity. SIAM J. Math. Anal. 10 (1979), 365-388.

[9] Clément, Ph.; Nohel, J.A.: Asymptotic behavior of solutions of nonlinear Volterra equations with completely positive kernels. SIAM J. Math. Anal. 12 (1981), 514-534.

[10] Dräger, J.; Klafter, J.: Strong anomaly in diffusion generated by iterated maps. Phys. Rev. Lett. 84 (2000), 5998-6001.

[11] Duoandikoetxea, J.; Zuazua, E.: Moments, masses de Dirac et décomposition de fonctions. C. R. Acad. Sci. Paris Sér. I Math. 315 (1992), 693-698.

[12] Eidelman, S. E.; Kochubei, A. N.: Cauchy problem for fractional diffusion equations. J. Differ. Eq. 199 (2004), 211-255.

[13] Feller, W.: An introduction to probability theory and its applications. Vol. II. Second edition. John Wiley \& Sons, Inc., New York, 1971.

[14] Grafakos, L.: Classical and modern Fourier analysis. Pearson Education, 2004.

[15] Gripenberg, G.: Volterra integro-differential equations with accretive nonlinearity. J. Differ. Eq. 60 (1985), $57-79$.

[16] Gripenberg, G.; Londen, S.-O.; Staffans, O.: Volterra integral and functional equations. Encyclopedia of Mathematics and its Applications, 34. Cambridge University Press, Cambridge, 1990.

[17] Ignat, L. I.; Rossi, J. D.: Decay estimates for nonlocal problems via energy methods. J. Math. Pures Appl. 92 (2009), 163-187.

[18] Jakubowski, V. G.: Nonlinear elliptic-parabolic integro-differential equations with $L_{1}$-data: existence, uniqueness, asymptotics. Dissertation, University of Essen, 2001.

[19] Kilbas, A. A.; Saigo, M.: H-transforms: Theory and Application. CRC Press, LLC, 2004.

[20] Kilbas, A. A.; Srivastava, H. M.; Trujillo, J. J.: Theory and applications of fractional differential equations. Elsevier, 2006.

[21] Kochubei, A. N.: Distributed order calculus and equations of ultraslow diffusion. J. Math. Anal. Appl. 340 (2008), 252-281.

[22] Kochubei, A. N.: Fractional-order diffusion, Differential Equations 26 (1990) 485492.

[23] Kochubei, A. N.: General fractional calculus, evolution equations, and renewal processes. Integr. Equ. Oper. Theory 71 (2011), 583-600.

[24] Ma, Y.; Zhang, F.; Changpin, L.: The asymptotics of the solutions to the anomalous diffusion equations. Comput. Math. Appl. (2013).

[25] Meerschaert, M.M.; Nane, E.; Vellaisamy, P.: Fractional Cauchy problems on bounded domains. Ann. Probab. 37 (2009), 979-1007.

[26] Metzler, R.; Klafter, J.: The random walk's guide to anomalous diffusion: a fractional dynamics approach. Phys. Rep. 339 (2000), 1-77.

[27] Metzler, R.; Klafter, J.: The restaurant at the end of the random walk: recent developments in the description of anomalous transport by fractional dynamics. J. Phys. A 37 (2004), R161-R208. 
[28] Nakagawa, J.; Sakamoto, K.; Yamamoto, M.: Overview to mathematical analysis for fractional diffusion equations new mathematical aspects motivated by industrial collaboration. Journal of Math-for-Industry 2 (2010A-10), 99-108.

[29] Nash, J.: Continuity of solutions of parabolic and elliptic equations. Amer. J. Math. 80 (1958), 931-954.

[30] Prüss, J.: Evolutionary Integral Equations and Applications. Monographs in Mathematics 87, Birkhäuser, Basel, 1993.

[31] Quittner, P.; Souplet, Ph.: Superlinear parabolic problems. Blow-up, global existence and steady states. Birkhäuser Verlag, Basel, 2007.

[32] Saloff-Coste, L.: Aspects of Sobolev-type inequalities. London Mathematical Society Lecture Note Series 289, Cambridge University Press, 2002.

[33] Schiessel, H.; Sokolov, I. M.; Blumen, A.: Dynamics of a polyampholyte hooked around an obstacle. Phys. Rev. E 56 (1997), R2390-R2393.

[34] Schilling, R.; Song, R.; Vondracek, Z.: Bernstein functions. Theory and applications. Studies in Mathematics 37, De Gruyter, Berlin, 2010.

[35] Schneider, W. R.; Wyss, W.: Fractional diffusion and wave equations. J. Math. Phys. 30 (1989), $134-144$.

[36] Sinai, Y. G.: The limiting behavior of a one-dimensional random walk in a random medium. Theory Probab. Appl. 27 (1982), 256-268.

[37] Triebel, H.: Interpolation Theory, Function spaces, Differential operators - 2nd, rev. and enl. edition. Johann Ambrosius Barth Verlag, Heidelberg, 1995.

[38] Uchaikin, V. V.: Fractional derivatives for physicists and engineers. Volume I Background and Theory. Nonlinear Physical Science, Springer, Heidelberg, 2013.

[39] Vazquez, J. L.: Barenblatt solutions and asymptotic behaviour for a nonlinear fractional heat equation of porous medium type. J. Eur. Math. Soc. 16 (2014), 769-803.

[40] Vergara, V.; Zacher, R.: Lyapunov functions and convergence to steady state for differential equations of fractional order. Math. Z. 259 (2008), 287-309.

[41] Vergara, V.; Zacher, R.: Optimal decay estimates for time-fractional and other non-local subdiffusion equations via energy methods. SIAM J. Math. Anal. 47 (2015), 210-239.

[42] Zacher, R.: A De Giorgi-Nash type theorem for time fractional diffusion equations. Math. Ann. 356 (2013), 99-146.

[43] Zacher, R.: Boundedness of weak solutions to evolutionary partial integro-differential equations with discontinuous coefficients. J. Math. Anal. Appl. 348 (2008), 137-149.

[44] Zacher, R.: Maximal regularity of type $L_{p}$ for abstract parabolic Volterra equations. J. Evol. Equ. 5 (2005), 79-103.

[45] Zuazua, E.: Large time asymptotics for heat and dissipative wave equations. Manuscript available at http://www.uam.es/enrique.zuazua, 2003.

Jukka Kemppainen, Department of Mathematical Sciences, Pentti Kaiteran katu 1, PO Box 3000 , FI-90014 University of Oulu, Finland, e-mail: jukemppa@paju.oulu.fi

Juhana Siljander, Department of Mathematics and Statistics, University of Jyväskylä, P.O. Box 35, 40014 University of Jyväskylä, Finland, e-mail: juhana.siljander@jyu.fi 
Vicente Vergara, Departamento de Matemáticas, Universidad de La Serena, La Serena, Chile, and Dirección General de Investigación, Universidad de Tarapacá, 1520 Arica, Chile, e-mail: vvergaraa@uta.cl

Rico Zacher, Ulm University, Institute of Applied Analysis, 89069 Ulm, Germany, e-mail: rico.zacher@uniulm.de 